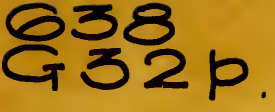




Digitized by the Internet Archive in 2015 


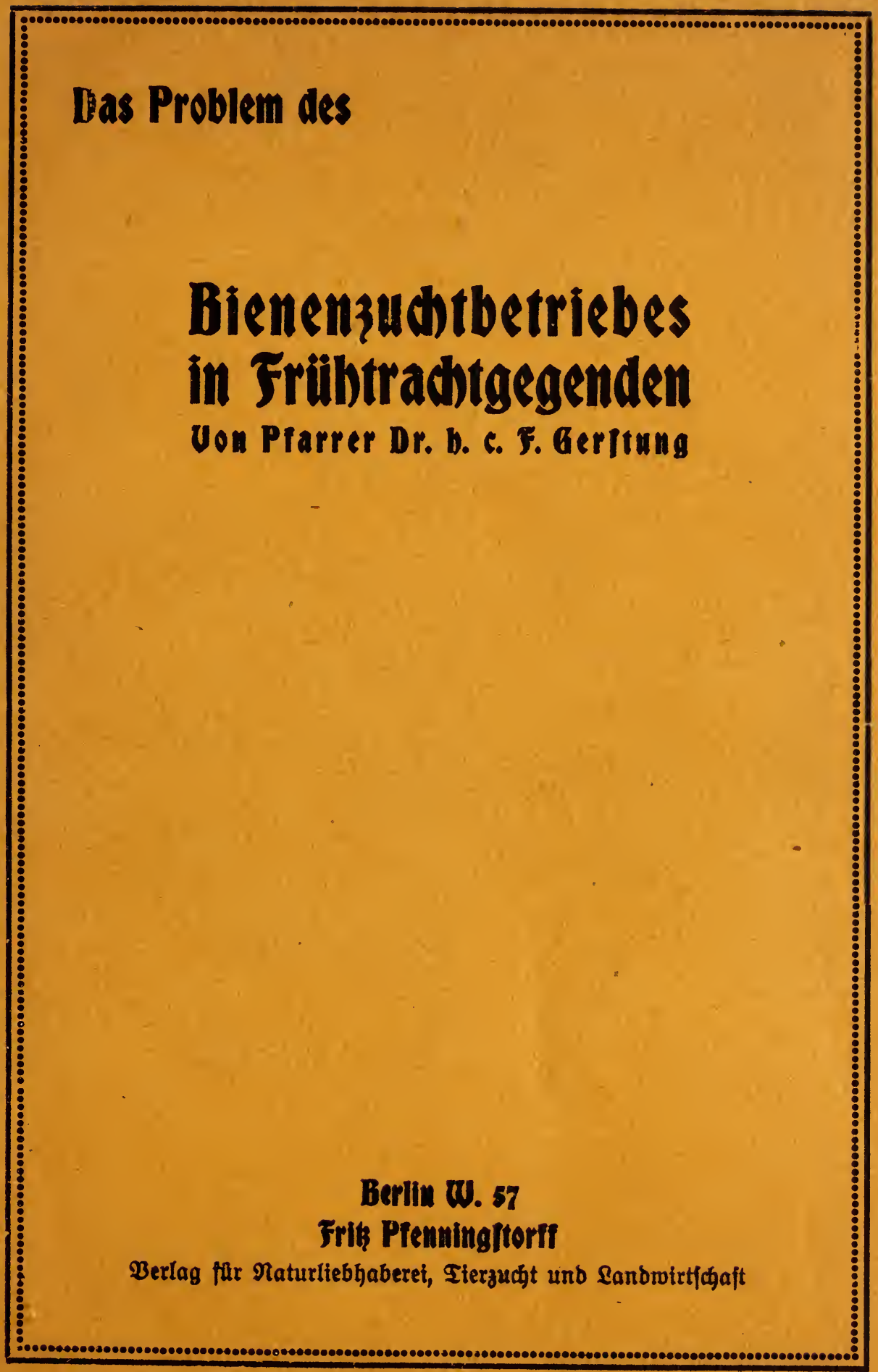




\section{Friz Pfenningitorff, $\mathfrak{B e r l i n} \mathfrak{W} .57$}

Berlag futr Naturltebhaberet, Tterzucht und Lanbwirtichaft

Poitt他eckonto: Berlin 39359.

Steinmebitraje 2.

\section{Gerbitneuigkeiten 1920:}

\section{Uniere Bienen}

Cin ausführliches Sanbbud ber Bitenenkunde und Bienenzudit. Serausgegeben von $\$$ farrer $\& u$ o w $i$. Un gefähr 700 Seiten mit zahlr. Tertabbilbungen unb 20 farbigen und fhwarzen Bilbtafeln auf Runftbruchpapier. Teil II: Bienenzucht. 420 Seiten mit einer Farbentafel, 6 Echwarztafeln und 303 Terts auntoruchpapter. Tetl Teil I exicheint porausfichtlic Enbe Dktober. Preis in einem Ganzleinenband gebunben 45 Mlk., in grei Sanzleinenbänben gebunben $50 \mathrm{Mk}$. Spätere \$reiserböhungen vorbebalten.

\section{Inhaltsulberitut:}

\section{T. Bienenkunbe.}

Die 2 ebeutung Der Bienenzucht: 1 . volks= mirtichaftliche Bebeutung; 2 . Bebeutung für Eanbwirtichaft, $D b f t=$ und Bartenbau; 3. wifferifiaftliche Bebeutung; 4. Bienenzucht als Rebensberuf und Nebenbef häftigung; 5. Bienenzudit als eble Liebhaberei.

Vorausjegungen für eine gebeihliche Bienentuht: 1. Bienenteide; 2. Rlima und $\mathfrak{W e t t e r ;}$ 3. Der Smker.

Die Naturgej hichte ber Bienen: 1. Bers

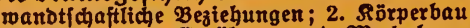
Der OBtenen; 3. Crnährung; 4. Wactsbau; 5. Fortp flanzung, Bererbungsgéése unto Raffenzucht; 6. Sirankheiten; 7. Feinde Der Biener.

Der Bien.

Unjere Bienen unb inre (bejuidute.

Dite Siteratur iiber unjere Bienen.

Unjere Bienen in Didtung, Sitte unb Volksglauben.

Bienenrecht.

\section{Bienenzudit.}

Die Bienenwohnung: 1. theoretif he Grunblagen F. D. Bienen wohnur sfrage; 2. Bauftoffe für bie Bienenwohnung; 3 . alts eingefilhrte 20hnungsarten; 4 . neue Erfindungen Der legten Sahre; 5 . bewegliche $2 B a b e ; 6$. Ab bergang von ber unberveglidien

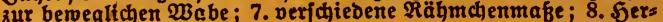
itellung ber $\mathfrak{B i e n e n v o b n u n g , ~ B a u ~ v o n ~ B i e n e n h a ̈ u f e r n ; ~ 9 . ~ थ ै u f s ~}$

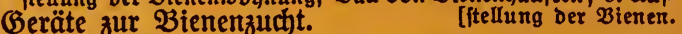
Bitenenpflege: 1. Umgan mit Bienen; 2. 2uswinterung;

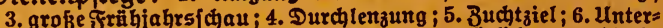
fiijbung und Semmung Der Triebe; 7. natiurlidie und künits liche $\mathfrak{B e r m e h r u n g}$ und $\mathfrak{P}$ flege Der Sunge und 2 uttervölker; 8. Sonigernte; 9. Wanderbienenzudt; 10 . Röniginnerzucht; 11. Drohnenzucht, 12. grofe Serbitichau; 13. Winterarbeit.

Erzeligntife ber Bitenentudt und thre Berwertung: 1. Qn und Berkauf und Berfand von Bienen; 2. Berkauf Des 5onigs; 3. 2Bert und Berwendung des Sonigs; 4. Uuslaifen Des $2 B a c h$ es: 5. WBachsverwertung im Broßbetrieb; 6. Sers ftellung ber Mittelwarb; 7. Fälihung und Unterfuchung yon

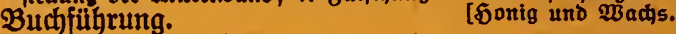
Die Erlernung Der Bienenzucht: 1. Einzelunterridt; 2. Eehrgang; 3. Smkeridule.

Das Jmkervereinswejen unb jeine 2ufgaben: 1. 2us gemeines; 2. Uusitellung; 3. פ⿴ufterfabung.

\section{Smkers}

\section{Jahr $=\mathfrak{u n d}$ Taj(d)}

ca. 250 Eetten, geb. Ralendaxium mit wagftodatabellen. 60 Eeiten Rotizblätter mit Tageseinteilung. Freie Blätter. Einnałmes uno थuss gabetabellen. Ubrefientafeln. 2rnweifungen für bie Mlonatsarbeiten. Das Bienenjahr. aberficht ber Blütebauer Der widhtigften Sonigs und Pollentrachtpflanzen. Trachtweijer. Saupttrachttabelle. Rähmchenmafe unb ihre

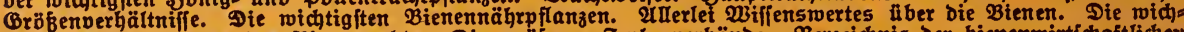
tigiten $\mathfrak{B}$ eftimmungen Des Bienentechts. Die gröberen $\$$ mkerverbände. Berzeichnis ber bienterwirtichaftlichert

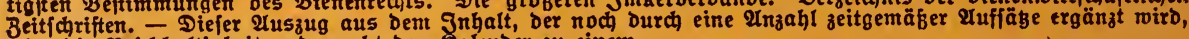
zeigt bie Reidbaltigkeit und macht ben salender zu einem

\section{auberorbentlid praktijacn Notiz= und Nadjiajlagebud,}

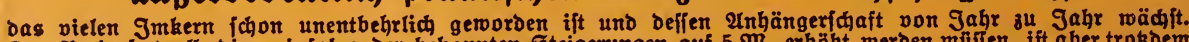

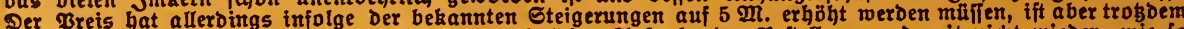

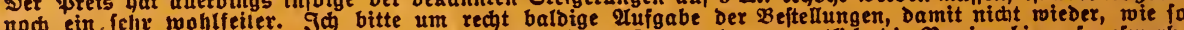

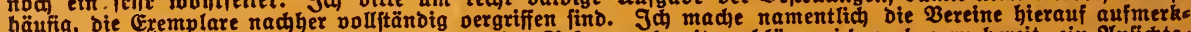

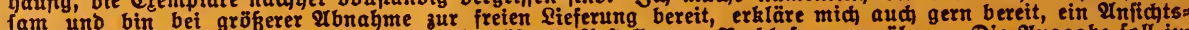

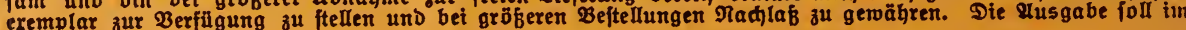
november exfolgen.

\section{Bereits vorliegente Neutgkeiten Des Sahres 1920.}

Brobe ifluftrierte Bienenflora Mittels europas. Dit Berlidfidtigung ber eins geführten Sulturgemädje it itberfichtridjer Form bargeftellt und mit einer sinleitung unb zahlreiden 2 bbilbungett, barunter einer Jarbtafel, verieben von Dtto Dengg. Bolls itălloig in 2 Teilen. \$reis für Teil I $28 \mathrm{M}$. Teil II erjøeint vorausfichtlid im Jabre 1921.

Sodh, Sarl, Berlin=Rantwik. Das Bicnen= wejen und bie Btenenplege. Stubient unb Erfahrungen. Umfang runb 100 S. Breis 4 Mit.

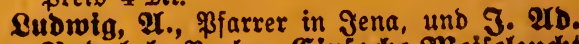
Rubolph, Burla. Einfadje Wetfelzudjt iür jebermann. Dit 19 Abb. \$reis $2,-$ Dit. Wetppl, Th., Der Bau bes Bienenhaujes. 3. 2uflare. Mnit 79 2เbb. \$reiß 6,- ylt. Weippl, Th., Wie bie Bienen einmal eine Republik maditen. Eine Befdid)te für jebermann erzäblt volt einent altelt sienen = freunde. Reu kerausgegeben. \$reis 75 Pfg.

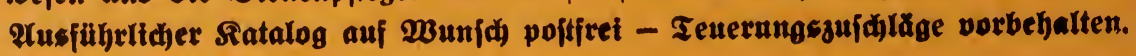




\title{
Das Problem
}

\section{des \\ Bienenjuchtbetriebes \\ in Srühtrachtgegenden}

\author{
Don \\ Pfarrer Dr. h. c. ferd. Berftung
}

Motto:

Fahret auf bie $F_{0 ̈ h e}$ und toerjet eure Nege aus, bá̉ thr einen 3 ug tut. \&uc. 5,4.

\section{Berlin W. 57}

Frits Pienningitorff

Verlag für Naturliebhaberei, Tierzucht und Lanbrittīaajt 



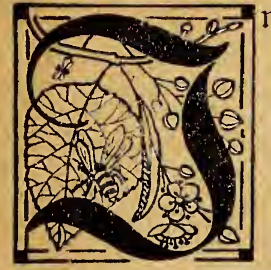

ausgeiprochenen Frühtrachtgegenden, in welchen die Bienenmeide fpätejtens Ende Juni ihren Sjöhepunkt er= reicht und bald barauf bie fchnell abjteigende Entwicklung eintritt, fo bá bann bie Tracht kaum zur Erhaltung Des Biens noch zureicht, befindet fich ber Bienenzüchter in einer fehr ïblen und kritifchen Lage, von welcher alle bie Jmker fich gar keine $\mathfrak{B}$ orjtellung machen können, bei benen bas Rnoblauch'iche $\mathfrak{B}$ erslein Geltung hat: „Wo Sonig umflutet bas Bienenhaus, ba heilen die grö̈bten Dummbeiten aus."

Diefe üble, krittiche Lage bejteht aber Darin, Daß̉ ber Frühtrachtimker

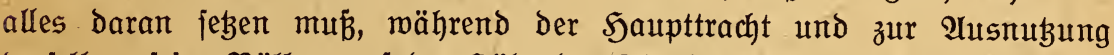
Derjelben feine $\mathfrak{V}$ ölker auf ber S̈̈̈he ber Flugkraft zu haben und jie bann

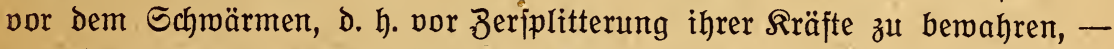
und dá̉ bann bie grof̉en Majien Bienenfleifch, bie er notgedrungen er= zeugen muk, ihm unmittelbar nach ber Saupttracht als "unnïbe Frefier" ericheimen, bie bem $\mathfrak{B}$ olke bie Winternahrung megzehren, ohne noch etwas zur Erhaltung bes Biens ober zur Erhöhung Der Ernte beizutragen.

Uus biejer offenbar höchit kritijchen \&age fint nun eine ganze Reihe von Betriebsweijen hervorgegangen, weldhe dem übeljtande gründlich abhelfen wollen, D. h. welche bie SJaupttracht mit ber SJauptmajie Flugbienten aus= mugen, bann aber auch zugleich bie $\mathfrak{B}$ ölker vor $\mathfrak{E r}_{\text {zeugung }}$ von unnübem Bienenfleijch bemahren mollen. Wir jagen nicht zuniel, wenn wir be= haupten, Dá̉ allen fcharfitchtigen Jüchtern in Frühtrachtgegenden ohne Spätiracht dies als bas für den Erfolg ausfchlaggebende Bienen= zuchtbetriebsproblem ericheint. •

Schon ber Altmeijter Dr. Dzierzon hat das Problem recht erkannt und es burch feine "biamantene Regel" zu löjen gejucht, indem er während ber Saupttracht bie Rönigin einfach in einen Räfig einjperrte, Damit fie längere Zెeit keine Eier legen konnte. Uuf biefe freilich recht barbarifche Weije wurbe ia bie Erzeugung von Bienenfleifch zur Unzeit ficher verbütet, aber Dr. Dzierzon hat auch fchon erkannt, baß̉ biejes $\mathfrak{B e r}=$ fahren mancher feiner bejten Röniginnen Das Seben gekojtet hat, ober baß̉ fie menigitens an ihrer Reijtungsfähigkeit (Einbußje exlitten hat und - was noch fchlimmer ijt, - bá baburch im Bienenvolke eine \&ücke entjteht, bie fich in ber Folge als höchit verberblich erwies. Es fehlten gerabe bie jungen Bienenglieder, melche Den Winter überdauern und als Flugbienten die erjte Frïhtracht im folgenden Jahre befliegen follten. Dieje höchjt gefähr= 
liche Qücke trat zumeijt in ihrer ganzen Wirkung gerabe in Der Zeit heroor, in welcher bie Grundlage für Den Erfolg im nächiten Jahre gelegt werden mußste, im zeitigen Frïhjahr, in welchem zum Schrecken Des Bienenvaters plößlich bie Bienen in Den $\mathfrak{B}$ ölkern wie Märzenjchnee durch bie Sonne verichwanden und ein vielleicht fichon umfangreiches Brutneft bienenleer zurücklteß̧en.

UlIs Serausgeber einer meitwerbreiteten Bienenzeitung wiffen wir aus Dreißjigiähriger Erfahrung, daßj in jedem Frïhjahr zahlloje Rlagen ange= jtimmt werden über Den auffälligen und Den meijten Jmkern unerklärlichen Rü̈kgang Der $\mathfrak{B}$ ölker gerabe in ber Zett, Da bie erjten $\mathfrak{B} l u ̈ t e n$ in üppigiter Fülle ihre Relche öffnen und Der Tijhh für bie Bienen in reichiter Weije gedeckt ijt. Da klingen bie Fragen über bie Urjachen folchen Rückganges und bie Bitten um Ungabe von Mitteln zur Ubhilfe ganz verzmeifelt. Die Mehrzahl Der Jmker macht Dann ganz unjchuldige und zufällige Tat= fachen verantwortlich, z. B. Das Blühen Der 2lpfelbäume, ober bes Weiß̄= Dorns, oder bes Flieders, jede Gegend hat ia in biejer Simficht einen bejonderen "Sündenbock", Der an Dem Bienenjterben fchuld fein foll. Die wenigiten fucten ben Grumb Da, wo er wirklich liegt, nämlich in bem 2rbjperren Der Rönigin in Der SJaupttracht im Jahre vorher, ober in ber Tatjache, baß̉ ber Smker zuviel alte, abgelebte und zu menig junge Bienen in Den Winter genommen hat, was eben auch eine naturgemäß̉e Folge

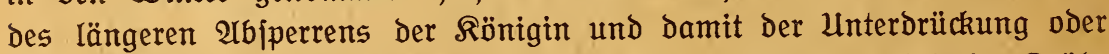
Befchränkung Der Bruttätigkeit ijt, ober in ber trachtarmen Zeit im Früh= herbjt, in welcher die Bruttätigkeit von jelbit, freilich viel zu früh für Das

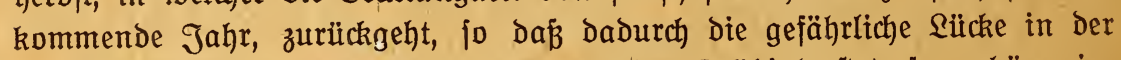
Bolksglieberung entiteht, welche im folgenden Frühjahr ftets jo verhängnis= voll wirb.

Die alte Gchule hat ja bieje Tatjache nicht leugnen können und als fie klar erkannt worben war, hat fie auch in ber fogenannten ipeku= lativen Sjerbitfütterung Das Mittel entbeckt, bieje Sücken Durch einen künjtlich hervorgerufenen, fpäten Sjerbitbrutjał wieder auszufüllen, freilich nicht immer mit Dem erwünjchten Errfolg, Da ja gerabe bie Rücke in Der Brutentwicklung Durch 2lbiperren Der ßönigin und Brutbejchränkung in ber Saupttracht einen Mangel an jungen Brutbienen zur Folge hatte, welche bie $\mathfrak{B}$ orausjebung Des Gelingens der jpekulativen Sjerbitfütterung find.

Weiterhin ging bie "Diamantene Regel" von ber grundverkehrten Unnahme aus, Daß̧ alle Bienen alle Tätigkeiten inmerhalb und außerbalb Des Stockes verrichten könnten, und baß̉ bei abnchmender ober ganz fehlender Brut auch bie jogenannten Brutbienen bie Tracht mit befliegen und Dadurch eine Erhöhung Des Sjonigertrags erzielt werden könnte. In Wirklichkeit ift es bagegen jo, daß̉ Die offene Brut gleichjam bie \$eitjche ift, bie bie Bienen' zur Entfaltung Des höchjten Sammeleifers antreibt und 
Daß̧ mit abnehmender $\mathfrak{B}$ rut auch ber Sammeltrieb erlahmt, wie bies am beften im Frühherbit in Die Ericheinung tritt, mo die brutleeren $\mathfrak{B}$ ölker auch bei guter Tracht und günjtigem Wetter mur jehr träge fliegen, während Stöcke mit jungen Röniginnen und jtarkem Brutjab noch emjig ichaffen.

Nachjem einjïbtige Jmker alle Mängel ber "biamantenen Regel" Dr. Dzierzons burchichaut hatten, ijt jie je länger je meniger zur 2lnwendung gekommen und heute bürfte jie in ber uriprünglichen Form und Durch= führung nicht mehr vorgenommen merben. Uber um jo mehr hat man fich nach anderen und bejieren Methoden umgejehen, um bas immer wieber und immer fchärfer fïch auforängende $\mathfrak{P r o b l e m ~ z u ~ l o ̈ i e n . ~ - ~ B r a c h t e ~ e s ~ d o c h ~}$ bie Einfühnung bes intenjiven landwirtjchaftlichen Betriebs, welche fich in der zweiten Sä̈lfte des vorigen Jahrhunderts in Deutichland vollzogen hat, mit jich, bá̉ überall die frïher vorhandenen डpättrachten verjchmanden und fich mindejtens $4 / 5$ des ganzen Gebietes nach und nach, aber unaufhaltjam, in Frühtrachtgegenden vermandelte. 2Uuch Die $\mathfrak{V e r k o p p e l u n g ~ D e r ~ F l u r e n ~}$ (Separation) hat ein gut Teil hierzu beigetragen. So kam es, dá̉ bas Problem Des Bienenzuchtbetriebs in Frühtrachtgegenden immer mehr in den $\mathfrak{V}$ ordergrund trat und eine befriedigende \&öjung erforderte. (Es ijt I

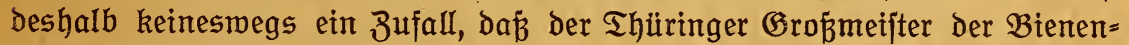
zucht, v. Berlepich, fein \&ehrbuch ausdrücklich jchon für bie Bienenzucht in Frühtrachtgegenden gejchrieben hat.

Baron $\mathfrak{v}$. Berlepich hat freilich bas Problem noch nicht jcharf genug erfapht. Er behandelt dasjelbe in Rap. XXXVII jeines Rehrbuchs und kennt mur auker ber ipekulativen Frühjahrsfütterung, welche er empfiehlt, bie fich aber längit als ein zweijchneibiges Schwert erwiejen hat, bie $\mathfrak{B}$ er ei $n i \mathrm{~g} u \mathrm{ng}$ der $\mathfrak{V}$ ölker im Sjerbjte, um möglichjt volkreiche Stöcke zu überwintern, welche imjtande find, bie erjte reiche Frühtracht aus Raps und Dbjtblüten auszumuken. Dabei kommt v. Berlepich nicht einmal ber Benanke, dá̉ zwei vereinigte $\mathfrak{V}$ ölker mit vornehmlich alten Bienen keineswegs volks= ftark ins Frühjahr kommen, jondern mur im $\mathfrak{B i n t e r ~ e i n e n ~ b o p p e l t j t a r k e n ~}$ Urbgang alter Bienen, aljo jtarken Reichenfall haben werden.

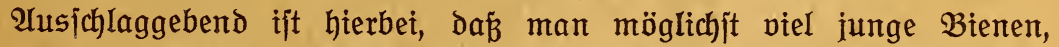
melche im Serbjt ihre Triebkraft noch nicht verbraucht haben, in ben Winter bringt, und welche dann auch bis zur Frühtracht lebensfähig bleiben.

Der berühmte Schüler des Baron v. Berlepich, $\mathfrak{W}$. (Günther in Bispers= leben, hat wiederholt nackte $\mathfrak{V}$ ölker aus ber Sjeide kommen laffen, um jeine infolge mangelnder Spättracht und Blütenmangel jchwachen $\mathfrak{B}$ ölker für den Winter aufzubefiern und für Das kommende Jahr jtark zu machen, aber er ijt zumeijt dabei Durch die Erfahrung belehrt morden, dá̉ dieje veritärkten $\mathfrak{V}$ ölker im kommenden Frühling bei ber 2Uuswinterung und erjt recht in ber erjten Saupttracht auch nicht ftärker waren, als bie nicht aufgebejijerten. Dá̉ Der (Brund für dieje Erjcheinung darin lag, bá̉ er 
Völkern mit viel alten abgelebten $\mathfrak{B i e n e n ~ n o c h m a l s ~} B$ ienen als $\mathfrak{B e r f t a ̈ r k u n g ~}$ beigegeben hatte, bie zum Teil auch ichon ihr Triebleben ausgelebt hatten, ijt ihm freilich auch nicht klar zum Bemubtjein gekommen.

Der verjtorbene Eijenbahnjekretär Schröber in Frankfurt a. $\mathfrak{M}$. hat in Den 80 er Jahren bes vorigen Jahrhunderts fich theoretijh und praktijch ernjtlich mit ber \&öjung Des Problems abgemüht, freilich hatte er nur bie eine Sälfte jharf ins 2 uge gefajł, welche auch $\mathfrak{v}$. Berlepich, wie joeben gezeigt, Durch $\mathfrak{B o ̈ l k e r v e r e i n i g u n g ~ f u ̈ r ~ g e n u ̈ g e n d ~ j t a r k e ~} \mathfrak{V}$ ölker im Frühjahr zu forgen, zu löjen verjucht hatte. Die andere Seite des Problems: $\mathfrak{B} e=$ jchränkung ber $\mathfrak{B} \mathfrak{r} u t$, um bie Erzeugung unnüber Frefjer zu verhüten, hat er nicht behandelt. Schröber tritt bafür ein, die Ent= wicklung Der $\mathfrak{B o ̈ l k e r ~ n i c h t ~ z u ~ j t o ̈ r e n , ~ a u c h ~ b i e ~ B r u t e n t w i c k l u n g ~ m a ̈ h r e n d ~}$ ber Şaupttracht nicht zu bejchränken, Da bas eine Beeinträchtigung bes Sammeleifers in jich jchlié̉en würde, wohl aber ftets bie fallenden $\mathfrak{V}$ or= jchnärme in einem Fach unmittelbar neben bem Mutteritocke aufautellen, in biejem Fach bauer und brüten zu lafjen und im Frühberbit diefe $\mathfrak{V}$ or= ¡chwärme nach 2lbtöten ber alten Rönigin mit Dem nachbarlichen Mutter= jtocke, welcher inzmijchen eine befruchtete junge Rönigin erhalten hat, zu I vereinigen.

Dies $\mathfrak{B e r f a h r e n , ~ w e l c h e s ~ u n s ~ u n j e r ~ v e r j t o r b e n e r ~ F r e u n d ~ a u s f u ̈ h r l i c h ~}$ mündlich geichildert hat, und worauf wir aud wiederholt empfehlend hin= gemiejen haben, hat Den $\mathfrak{V}$ orzug, Daßa die SJaupttracht mit Der SJauptmacht Der Flugbienen ausgenugt wirb, ohne bá̉ Der freien Entwicklung Der $\mathfrak{V o ̈ l k e r ~ j e l b j t ~ b i s ~ z u m ~ S c h m a ̈ r m e n ~ i r g e n d e i n e ~ S c h r a n k e ~ g e j e g t ~ m i r d , ~ w e i t e r h i n , ~}$ Dá̧ auch die dem Schwarme innewohnenden ftarken Entwicklungskräfte vollauf ausgenugt merben, bá̉ aljo von ber Triebkraft bes $\mathfrak{B}$ olkes nichts verloren geht, unterbrückt mird ober unausgenubt bleibt und fchließjlich, Dả Das Muttervolk mit junger Rönigin, Der Grundlage ber bejten Ent=

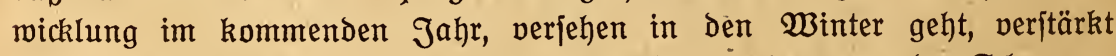
Durch bie zahIreichen ju $\mathfrak{n}$ gen Bienen, welche aus dem von ber Schmarm= mutter heritammenden Brutnejt ausjchlüpjen.

Wir felbjt haben längere Zeit dieje Methode mit beftem Errfolge bei einem Teile unjeres Standes angemandt und hatten zu biefem Jwecke unjern 42 fächerigen Pavillon jo hergeitellt, Daß alle Beuten untereinander Durch $\mathfrak{B e r e i n i g u n g s l o ̈ c h e r ~ v e r b u n d e n ~ m a r e n . ~ D i e j e ~ B e t r i e b s w e i f e ~ l e i b e t ~ a b e r ~}$ an einem bedenklichen übelitande: Man mú̉ ftets die doppelte 2 nzahl

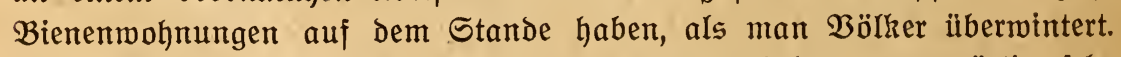
Das verteuert naturgemäß bie 2lnlage, zumal bei ben gegenmärtig jehr hohen Preijen, bedeutend und dürjte der Grund fein, weshalb fich bieje jonjt jehr empfehlenswerte Betriebsweije nicht in allen Frühtrachtgegenden eingeführt hat.

Sjätte Schröber - was mande Smker getan haben - bein Schroärmer 
Den 2 uutteritock um eine Stockbreite zur Seite gerückt und Den Schmarm an Der Stelle bes $\mathfrak{M} u t t e r i t$ tockes aufgejtellt, ober hätte er Den Mutteritock in bas Nachbarfach unmittelbar nach Dem Schwärmen umgehängt und ben Schwarm in Das Fach Des Mutteritocks eingeichlagen, fo baß̉ ihm alle Flugbienen bes Muttervolkes zufliegen muß̈ten, jo hätte Der Föriter Weide= mann jeinen "Frörteritock" nicht zu erfinden gebraucht, Denn biejer jtellt ja im Grunde genommen und uriprïnglich nichts bar als das Schröberiche Berfahren, angemandt auf unjere Thüringer Lagerbeute. Schröber imkerte noch in SHiterladern und bürfte Daher vor einem. Umbängen des Mutter= ftockes zurückgejchreckt fein. Wir halten es für bie Pflicht gejchichtlicher Wahrhaftigkeit, auf biejen Tatbejtand immer wieber hinzumeijen, um bie Ehre für bie erite Inmendung ber eigenartigen Betriebsweife bem zu= kommen zu laffen, dem fie gebührt, und dem abzuiprechen, bem fie nicht gebührt.

Ess wïrbe uns hier mun zu weit führen, wollten wir alle neuen Betriebs= weifen, welche im Grunbe genommen auf das Schröberjhe Zెmei=:Bolk= Syitem mit Umichalten der Flugbienen zum Borjchmarm und Wieder= vereinigen von Mutterjtock und Schmarm hinauslaufen, ausführlich be= handeln. Entichiedenen Wideripruch aber müffen wir erheben gegenüber allen Bejtrebungen, welche darauf ausgehen, für folche boch jehr einfeitige Betriebsweijen bejondere Wohnungsinjteme zu konjtruieren und dieje Dann als bie unübertrefflichjten und im Syonigertrag benkbar bejten auszupojaunen. Wenn wir für jede derartige bejondere Betriebsweije eine bejondere Bienen= wohmung heritellen wollten, fo würde bie Berwirrung auf bem Gebiete ber Bienenwohnungsfrage eine grenzenloje werden. Der Schwerpunkt in ber $\mathfrak{W}$ ohnungskonjtruktion und in Der Bewertung Derjelben barf boch nicht in ganz nebenfächlichen Dingen gejucht werben, fondern ftets in Dem Rardinalpunkt, ob die Wohnung für Den Bien die befte Entwicklungs= und Erhaltungstätte darbietet. Ulles andere find Fragen zweiter und britter Dromung, welche für bie Bewertung nie= mals ausfhlaggebend fein bürjen; wenn fie auch für den Betrieb manche Borteile und Erleichterungen bieten, es find nicht Lebensfragen für ben Bien, fondern Betriebsfragen für Den Imker.

Wir begnügen uns, Darauf hinzumeijen, dá̉ fchon ber Mönch $\Im u l i a n$

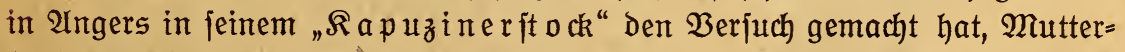
ftock und Schwarm, ja jogar mehrere $\mathfrak{B}$ ölker zu gemeinjamer 2Urbeit, aljo

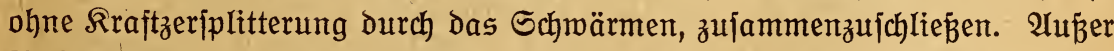
Weidemann verfolgt auch ber "Lambertzwilling" (hergeftellt von

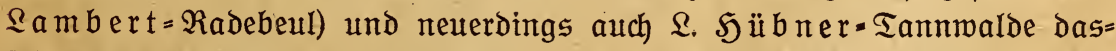
jelbe Ziel. Sebterer nimmt jogar an, daß̉ die immer wiederkehrende Brutbejchränkung zu eitrer Entartung Des Biens führen muß̌b, bie jich bei Der $\mathfrak{B}$ ererbung im $\mathfrak{B i e n}$ geltend macht. (Er warnt Daher vor ber jebt 


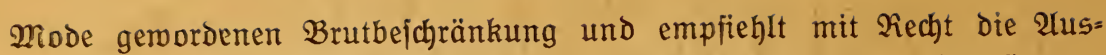
nubung ber Triebkräfte Des Biens, bie Doch vornehmlich in Der Empor= entwicklung bes Biens bis zum Sö̈hepunkt des Schmärmens in bie (Er= jcheinung treten.

In Dem von uns konftruierten, weitwerbreiteten Thüringer 3milling und Den Thüringer Einbeuten läß̈t fich jeberzeit, ohne viel an ihnen zu verändern, das Echröber'iche $\mathfrak{B e r f a h r e n ~ D u r c h j u ̈ h r e n . ~ U n j e r e ~ r a t i o n e l l e n ~}$ Beuten haben babei den $\mathfrak{B}$ orzug, Daß́ fie in eriter Sinie bie Entwicklung bes Biens begünjtigen und beförbern, Dann aber auch alle bie befonderen Wap̉nahmen geitatten und leicht und bequem gejtalten, welche nötig find, um alle Triebkräfte bes Biens Dem Imker bienjtbar łu machen, wie mir dies weiter noch nadjweifen werden. Wir halten $b a s$ für ben gröbten Vorzug der Thüringer Beuten, bá̉ fie mit höditer Bienen= gemäbheit bes Brutraumes als der Entmicklungs=und Er= haltungsfätte für ben Bien, größte (Einfachleit und $\widetilde{z}$ weck=

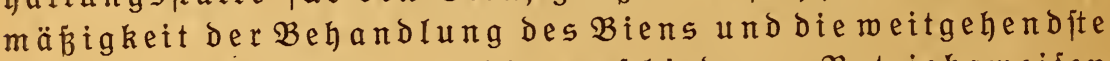
anpafingsfähigkeit an die verfaiedenen Betriebsmeifen verbinden und bamit ben fdimmiten Fehler, Bienenmiorig= kett auf ber einen Seite, und Rompliziertheit in ber Ron= ftruktion und einfeitige anpafiung an eine befondere $B e=$ triebsmeife auf ber anderen Seite ftreng vermeiden.

Der Erite, welcher Das Problem der Betriebsmeije in Frühtracht= gegenden icharf erfaß̧t und in eigenartig folgerichtiger Weije zu löfen ge=

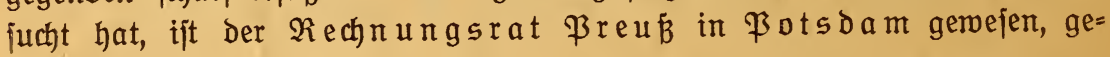
ftorben 1910.

(Es trifft fich auß̧erorbentlich günjtig, Daß̧, als wir mit ber Niederichrijt biejer arrbeit bejchäftigt waren, unter dem Titel "Die Preub́iche Imker= ichule", von ber Tochter Des Berftorbenen bie Betriebsweije ihres Baters aus hinterlaffenen 2 ufzeichnungen besfelben ausführlich bargeftellt und als III. Band Der "Bücherei für Bienenkunde" bei Theodor Fifher in Freiburg i. $\mathfrak{B r}$. peröffentlicht hat. Wer fich mit ber eigenartigen Betriebsmeife Preub' recht vertraut machen will, den verweifen wir baher auf biejes fehr lehr= reiche Büchlein. Der Sejer wirb nach Beendigung Der Sektüre jehr eritaunt fein über eine eigenartige Entbeckung. Orlles Wejentliche, was Runbich und andere als etwas Junkelnagelneues ber Deutichen Imkerwelt verkündet haben, ijt Der Preuß̉'ichen Betriebsmeije entlehnt. Nur Das „Drum und Dran", D. h. Das unwejentliche und Nebenjächliche ift von Runtich hirzugegeben worben, z. B. Die Dbenüberwinterung, Der Schlittenbetrieb und Dergleichen. Breuß̄ fchreibt: M2leine Betriebsmeife ift mur für Begenden ohne Spätfommertracht berechnet; fie ijt auch bisher ur in einer folchelt (Begend von mir praktifa) erprobt. Die brei leitenden Gefichtspunkte meiner Betriebsweije find folgende: 
1. In Begenden ohne Spätjommertracht können nur joldse Bölker etwas Iüchtiges leiften, die fhon bei Eintritt ber Frühommertracht fehr volkftark find.

2. Diefe jehr ftarken $\mathfrak{B}$ ölker müffen - und dies mödte ich für den fpringenden Punkt meiner Betriebsmeije er= klären - während der ganzen Trachtzeit oon Schrarm= gedanken abgehalten werben.

3. Mit Eintritt und während ber Sfaupttrachtzeit ijt ein übermäßiges $\mathfrak{B}$ rüten der $\mathfrak{B}$ ölker zu verhüten.

Rlarer und beftimmter kann man bas Ziel und die einzujhlagenden Wege in menigen Worten nicht ausjprechen und wir müjijen anerkennen, Dá̉ auch die ganze Durchführung Der eigentartigen Betriebsmeife äuß̉erj̣ folgerichtig bargejtellt ift unter fcharfer Berückfichtigung ber gegebenen Irachtverhältnifie.

Punkt 1 fucht er zu erreichen burch ipekulative Sjerbjtiütterung vom 1. bis 21. 2uguft; Punkt 2 burch Umbängen bes Brutnejtes in bie obere Etage und, Damit verbunden, Durch Ermeiterung Des Brutneftes in ber unteren Estage während bes Monats Mai; Bunkt 3 Durch Bejchränken Der Eierlage Der Rönigin in Der Zeit vom 20. Mai auf Drei Waben, in Der Jeit vom 21. bis 28. Wai auf zwei Waben und nach bem 28. Mai auf eine Wabe. Mit biefer Brutbejchränkung iłt ein 2rbjperren der Rönigin und ein fünfmaliger Wabenwechjel verbunden, welcher genau alle acht Tage, vom 10. Juni bis 12 . Juli vorgenommen wird und ben Zुweck hat, Die brutbefebten $\mathfrak{W a b e n ~ i n ~ D e n ~ S J o n i g r a u m ~ z u ~ h a ̈ n g e n , ~ D a f u ̈ r ~ a b e r ~}$ Der Rönigin neue, leere Brutwaben zu geben. - Nach Beendigung Der Iracht und 2ufhören ber Schwarmlujt wirb bie Rönigin wieder freigegeben. Für Erneuerung ber Röniginnen wirb burch bejondere Weifelzucht gejorgt.

Der ganze Betriebsplan ijt den Trachtverhältnijifen Durchaus ange= paß̉t, klar burchdacht und folgerichtig von 2lnfang bis zu Ende Durchgeführt. Man könnte ihn eine veredelte und verbefferte Form ber Dzierzon'jchen "Diamantenen $\Re$ egel" nennen, welche die Widernatürlichkeit ber Ein= iperrung Der Rönigin in einem engen Räfig vermeibet, Den Bruttrieb nie ganz unterbrückt und bamit ben Fleip̈ ber $\mathfrak{B}$ ölker möglichjt menig beein= trächtigt, das Echwärmen jicher verhütet und bas Erzeugen von honig= frefiendem Bienenfleifch zur Unzeit verhindert. - Dabei merkt man bei Der ganzen Durchführung Des Blanes, daß̧ ein kundiger Meifter, ber auch bie Rebensgejebe Des Biens völlig beherricht und jich dienjtbar zu machen veriteht, als spiritus rector, als führender (beijt bahinterfteht.

Wir müjfien anerkennen, dá̉ dieje Betriebswetje für ganz bejtimmte Trachtverhältnifie raffiniert zweckmäß̈ig ausgedacht ijt, und müjien es loben, dá̉ Preuß́ nicht fo, wie Runbich es leider getan hat, Der unter gleichen Berhältnifjen faft in gleicher Weije geimkert hat, dieje Betriebsweije für 
alle, auch für ganz anders geartete $\mathfrak{B e r h a ̈ l t n i f f e ~ e m p f o h l e n ~ h a t . ~} \mathfrak{E r}$ war

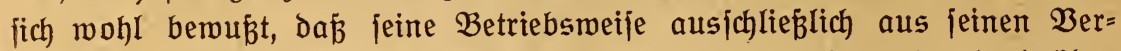
hältnififen ermachjen war und fich auch nur unter gleichen ober boch ähn= lichen $\mathfrak{B}$ erhältniffien bemähren konnte. Er warnt in ehrlicher Weije jogar vor unüberlegter und unbejonnener übertragung feiner Betriebsmeife auf anders geartete $\mathfrak{B e r h a ̈ l t n i f f e . ~}$

Wenn wir num kritijch zu biejer Preußj'ichen Betriebsmeije Stellung

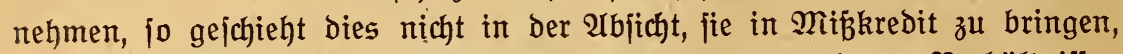
fondern hinzumeijen auf die Punkte, welche unter anderen' $\mathfrak{V}$ erhältniffen abgeändert werben müiffen, und vor allen Dingen, um zu zeigen, wie ber= felbe $3_{\text {meck }}$ auf einfachere und bienengemäß̈ere Weife erreicht werben kann.

$\mathfrak{W i r}$ bezweifeln, Daß̉ für die ipekulative Serbitfütterung vom 1 . bis 21. 2ugujt genügend junge $\mathfrak{B}$ rutbienen, von benen, auß̉er von ber Rönigin, Das Gelingen und der Errolg Der ipekulativen Sjerbjtfütterung, wie fchon oben gezeigt, abhängig ift, vorhanden find. Man mú̉ boch

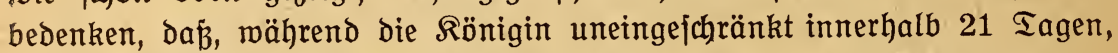
aljo innerhalb einer Brutperiobe etwa 50000 Bienen erzeugt, fie bei bem 2bjperrwerfahren auf eine Wabe in Derjelben Zeit höchitens brei Waben mit ie etma 5600 (Normalganzrahmen!), aljo 16800 junge Bienen liefert. Wirb ber "Wabenwechjel" jechsmal vollzogen, jo fehlen bann 33600 junge Bienen in zmei Brutperioden. Das kann bie freigegebene Rörigin in 14 Tagen nicht nachholen, ganz abgejehen Davon, bå̉ bann ichon bie $\mathfrak{a} \mathfrak{b}=$ ¡teigende Entwicklung begonnen hat. Wünfchenswert wäre es baher, wenn ein voller Julibrutjab auslaufen würde, melcher bie Bürgichaft für ben Erfolg der ipekulativen Serbjtfütterung bieten würbe. Das ijt jedoch bei dem Preußj'ichen Berfahren völlig ausgejchlofien. Die jehr günjtige, früh= zeitige Tracht in Der Potsdamer Gegend hilft bazu, Daß̉ bie Nachteile der entitandenen $\mathfrak{B}$ olkslücken nicht jo verberblich hervortreten, ba fichon im zeitigen Frühjahr infolge fehr günjtiger Trachtverhältniffe wieber eine ¡łärkere Brutentwicklung einjebt, 14 Tage früher etwa, als z. $\mathfrak{B}$. Durch= ichnittlich bei uns in Thüringen. Dies kann für bie 2 usmubung ber Eommertracht von ausjalaggebender Bedeutung fein, ba ja in allen Frühtrachtgegenden der $\mathfrak{P u n k t} 1$ von $\mathfrak{P r e u} \mathfrak{B}$ gilt, Daß̧ nur folche $\mathfrak{B}$ ölker etwas Tüchtiges leijten, bie fchon bei (Eintritt ber Frühjommertracht fehr volkjtark, b. h. auf ber Söbe ber Flugkraft iteh'en und bas ijt leiber eben nur in jolchen Gegenden zu erreichen, in Denen bie erjte Früh= lingstracht früh einjebt, jo dá̉ zmijchen ihr und ber Saupttracht wentigitens fünf Wochen bazwijchen liegen, da ja mur foldhe Bienen bie Tracht aus= nußzen, wie auch ßreú jehr richtig betont, welche 36 Tage vorher als Eier im Brutneft erichienen find. Da nun auch bei uns bic Syauptracht aus Der Ejparjette in günftigen Jahren, wie Das Jahr 1920 eins war, fchon vom 20. 2hai an einfebt, fo müßjten bie Eier für bie Bienen diejer Şaupt= 
tracht fpätejtens am 14. 2pril gelegt merben. In Diejer Zeit aber herricht hier zumeift noch recht bienenmibriges Wetter und Rälte und an Tracht ijit oft auch noch nicht zu Denken, vor allem fehlt bie Bollentracht, von welcher hauptjächlich die flotte Frïhjahrsbrutentwicklung abhängig ijt.

So aber ijt es in den meijten übrigen Frühtrachtgegenden Deutjchlands beitellt mit Uusnahme von jolchen, Die klimatijch günjtiger liegen, wie bie warmen Fluß̧täler des Rheins, des Mains ujw. WBir müfjen baher vom Serbit her jehr itarke $\mathfrak{B}$ ölker mit meijt jungen Bienen in die erite Frühtracht bringen, bamit während derfelben der

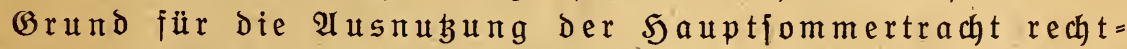
zeitig gelegt wirb. Wit anderen Worten: Der Erfolg unjerer Bienen= zucht ift zum groben Teil abhängig von Der Berfajiung ber $\mathfrak{B}$ ölker, melche

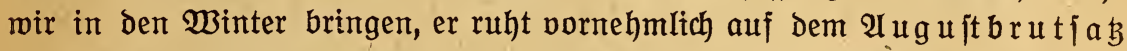
und Damit auf Der ipekulativen Serbitfütterung in jolchen Gegenden, in Denen Der Uugujt fchon trachtarm oder fajt trachtlos ift. Da mun aber Das Gelingen Der ipekulativen Sjerbitfütterung auß̧er von einer jungen fruchtbaren Rönigin hauptjächlich von bem $\mathfrak{B}$ orhandenjein zahlreicher j $u$ n g e r brüteluftiger Bienen abhängig ifit, jo mülijen mir jebe Bejchränkung Der Bruttätigkeit Der Rönigin in Der zmeiten Syälfte bes Juli verwerfen,

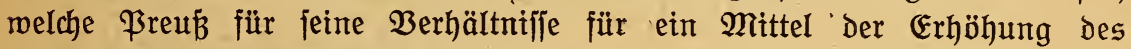
Sonigertrages betrachtet.

Wenn unter unjeren $\mathfrak{B e r h a ̈ l t n i f j e n ~ D a h e r ~ e i n e ~} B$ rutbejchränkung über= haupt $3^{\text {meck }}$ haben follte, fo hätte fie fünf Wochen vor Dem 1 . Jult ein= zuleben und 14 Tage anzuhalten, aljo vom 23. Mai bis 7. Juni jtatt= zufinden, ba in biefer Zeit die Bienen als Eier gelegt merben, welche anfangs Juli Flugbienen merben. Infangs Juli aber bietet gerabe in vielen Gegenden bie Sinde und auch ichon Der Weipklee reiche Tracht bar, io Dá̉ für jolche auch diefe $\mathfrak{B r u t b e j c h r a ̈ n k u n g ~ v o m ~ u ̈ b e l ~ m a ̈ r e . ~ D a ~} \mathfrak{s} \mathfrak{R}$ ezept,

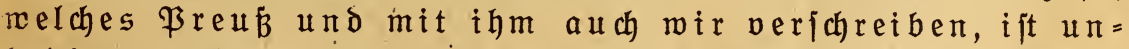
bedingt richtig, da B fünf $\mathfrak{B}$ ochen oor Eintritt trachtlojer Zeiten bie Erzeugung von unnübem Bienenfleifch verhütet werden múb, wenn bie Sonigerträgenicht gejchälert merben

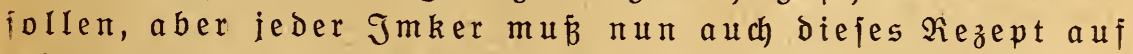
feine eigenartigen Trachtverhältnifje richtig anmenden. Ein ichablonenhaftes Befolgen besjelben ohne Rückjicht auf bie (Eigenart der Begend kann jogar die Urjache völligen Mīßerfolgs unter jomit günjtigen Bedingungen fein. Wir kommen darauf nochmals bei Bejprechung bes Punktes 3 zurück.

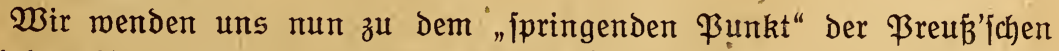
Betriebsweife: Unterdrückng ber $ऽ \mathfrak{d}$ marmgedanken während bcr $\mathfrak{S} a \mathfrak{u}$ ttrach t.

Uuch hier fimmen wir, wie wir ichon oft genug in Wort und Schrift 
erklärt haben, mit ஒreuś und allen anderen, die auf gleichem Standpunkte jtehen, völlig überein, da in Frühtrachtgegenden bekanntermaß̧en jedes Schwärmen vor oder während ber Sjaupttracht den Jmker um jeine Sjonig= ernte bringt. Zumeift merden burch bas Schrärmen nicht nur die Rräfte zeriplittert, jondern bie bejten Trachttage merben auch verjäumt und ichlie $\bar{B}=$ (ich) hat man anjtatt einer befriedigenden fonigernte zwei oder mehr Bölker, die nicht einmal ihren Winterjtand eingetragen haben. $\sigma_{\text {ch }}$ a $r m=$ betrieb und Frühtrachtgegend pafien wie die Fauft aufs a u ge zu einanber, b. h. fie ichließ̉en fich gegenfeitig aus. Man kann dies ben Frühtrachtimkern nicht oft genug jagen, da es immer noch viele gibt, die fich über jeden frühzeitigen Schmarm freuen, ohne zu bedenken, Dab́, menn alle $\mathfrak{B}$ ölker ihnen dieje Freude machen mürden, fie nie eine gute Sfonigernte erzielen würden.

Uuch mas Preuß̧ über die Einführung ichroarmluftiger Bienenrafjen, aljo ber Sjeide = und Rrainerbiene jagt, dą̧ dieje zum Ruin der Bienen= zucht in Frühtrachtgegenden führen können, unterjareiben wir voll und ganz und wir haben gleich ihm oft genug Dagegen unjere marnende Stimme

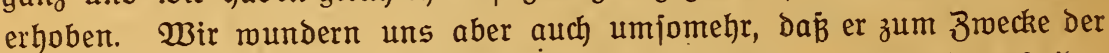
Bermehrung Der $\mathfrak{B}$ ölker fchließ̉lich doch die Einführung von nackten Sjeide= völkern empfitehlt!

Schon Der Urtmeijter Gravenhorjt hat auf die Wittel und, Wege hin= gewiejen, welche bas vorzeitige Schrörmen verhüten und die 1 usnubung ber Şaupttracht ermöglichen follen: Ermeiterung und $\mathfrak{a}$ seinander ziehen des Brutnejtes, Bauenlafien und bleichachen der Bölker rectzeitig oor Einjegen ber 5 ommertracht. Diejelben Mittel empfiehlt auch Preuß̧ und auch wir haben in unjerem Sehrbuch "Der $\mathfrak{B}$ ien und feine 3 ucht"-ausführlich dargejtellt, wie man den Schwarmtrieb im Zaume halten kann, ohne ber Entroicklung Des Biens irgend welche Schranken aufzuerlegen. In der Sauptjache läuft die $\mathfrak{B} e=$ handlung der Bölker vor und während ber Saupttracht Darauf hinaus, die nieberen Triebe, Den Urbeiterbruttrieb, den Bautrieb und unter $14 m=$ jtänden aud den Drohnenbruttrieb auf breitejter (Brundlage in 21njpruch) zu nehmen, um auf dieje Weije das Emporklettern Des Biens auf die höchite Eproffe der Entwicklung, auf die Schrarmböhe, entweder ganz zu verbüten oder möglichjt meit hinauszuziehen. Jeigen jich Durch alnblajen von Weifelzellen ober Durch auffällige Stockung in ber Bautätigkeit Doch

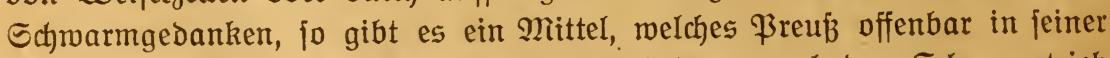
Wirkung noch nicht kannte, welches auch Dann nod) Den Schrvarmtrieb

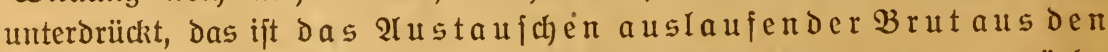
¡cmarmlujtigen $\mathfrak{B}$ ölkern gegen offene $\mathfrak{B}$ rut aus zurück= gebliebenen $\mathfrak{B}$ ölkern, modurch zugleich auch ein (Bleichmadjen vor

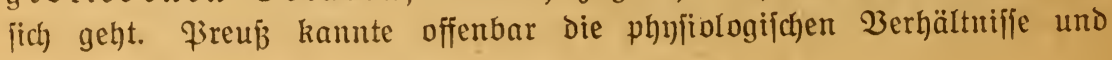


Sebensgejege Des Biens, auf Denen Die ganze Entricklung Des Biens be= ruht, nicht genügend, fonjt würbe er bie Schluß̧plgerungen baraus für bie ganze Bienentlege in Frühtrachtgegenden noch fchärfer und plan= mäß̈iger gezogen und angemandt haben.

Breuß ift ein Meifter in Der Darjtellung Der wohlüberlegten technijchen Durchfühnung ber Errweiterung Des Brutneites und auch bes Bleichmachens Der $\mathfrak{B o ̈ l k e r ~ - ~ a b e r ~ w e n n ~ i r g e n d w o , ~ j o ~ m e r k t ~ m a n ~ h i e r b e i , ~ d a ß j ~ b i e ~ E n t = ~}$ wicklung längit über ihn hinausgegangen ijt und zwar gerabe nach der

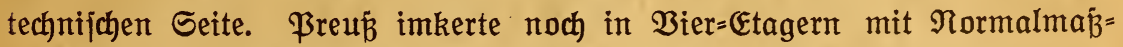
ganzmaben und Behandlung von hinten. Saraus erklären fitch bie grenzent= Iojen Schwierigkeiten und $\mathfrak{U}$ mitände und leider auch jchwerwiegenden $\mathfrak{B i e n t e n}=$ wibrigkeiten, welche mit jeiner Betriebsweije in biejer Entwicklungsperiode Des Biens verbunden find. Schon im Mai quellen inm jeine mit zrölf Normalmaben ausgejtatteten $\mathfrak{B}$ ölker hinten heraus und bie 2Ungit vor vorzeitigem Schwarmtrieb kommt über ihn und verläßjt ihn nicht mieder.

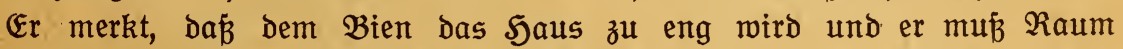
ichaffen für ben wachjenden Bien, der im Brutneft fich nicht mehr aus=

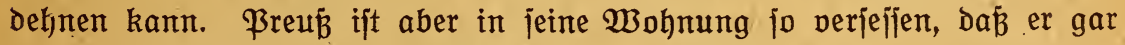
nicht merkt, dá̉ die bienenwibrige Form berjelben ihn zu allerlei ebenjo bienenwiorigen Maß̄nahmen zwingt, bie er gemí nicht ausgeführt hätte, wenn er in feiner $\mathfrak{W}$ ohnung einen anderen und befieren 2 usweg gefunden hätte. Ulm Das Brutnejt genügend erweitern zu können, hängt er Das uriprünglich in ber unteren Etage befindliche in ben Syonigraum und bie Rönigin febt er mit einer auslaufenden Brutwabe und einigen leeren, ausgebauten Waben oder auch Runitwaben und einem jogenannten $\mathfrak{B a u}=$ rahmen in bie untere Etage, welche nun Durch 2lbiperrgitter vom Sfonig= raum getrent wird und ebenjo vorn Durch 2bjperrgitterjated die Rönigin an etrwaigem $2 u s j c h$ wärmen hindert. Uuf bieje Weije wirb bas Brutnejt in zwei Teile zerrifien und damit bie Brutneftoronung gerabe in ber Periobe grïndlich gejtört, in welcher fich Der Bien ungehindert und jchnell bis zum Gipjelpunkt entroickeln joll. Schon burch die groß̉en Schwierigkeiten uno Die zeitraubende Durchführung bes Erweiterns und llmbängens der Brut= nejter in Beuten mit Behandlung von hinten bürften fich viele abhalten lajien, bie Preup'iche Betriebsweije einzuführen. Runbjich hat Durch (Ein= führung Der Breitwabe und Des Ulberti=Seitenjchteber=Snjtems in ber unteren Etage jeines 3 willings bieje Schwierigkeiten etwas zu erleichtern gejucht; behoben und bejeitigt hat er fie baburdh keineswegs, zumal auch jeine $\mathfrak{B}$ rutwabe und fein Brutraum für bie Entroicklung eines vollkräftigen Bolkes zu klein find. Weder Preuß noch Runbich nehmen, gezmungen burch ihre Stockformen, bei biejer Behandlung ber $\mathfrak{B}$ ölker in ber für ben Frfolg ausichlaggebenden Entwicklungszeit Rückjicht auf bie (G rundtat fache, dá̧ ber $\mathfrak{B} i e n$ gerabe in jeinem $\mathfrak{B r u t n e j t}$ eine wunberbare, ge= 
¡chlofiene Einheit baritellt, welche von den zmeckmäßigiten Sebens= und Entwicklungsgejeßen beherricht wird, Deren Störung ftets eine Beeinträch)= tigung und Echäbigung ber ganzen Entroicklung und bamit auch bes (Er= trages zur naturgemäß乃 Fon Folge haben mú̉.

Bienenmibrig ift por allem auch bas $\mathfrak{B}$ ermandeln bes bisherigen $B$ rut= neites in Den Sonigraum, mie dies burch Umbängen bet $\mathfrak{P r e u k}$, und auch bei Runbich Durch 2lbjtecken Der Rönigin in Der unteren (Etage gejchieht. Die Erfahrung lehrt, dá̧ gerade im zeitigen Frühjahr jehr viel Pollen rings um das Brutnejt herum abgelagert mird, welcher bie Baujteine für die Entwicklung ber Brut Darbietet, jobá̈ eine Trennung Des Pollens von Der $\mathfrak{B}$ rut eine verderbliche Unterernährung der $\mathfrak{B}$ rut zur Folge hat. Die nicht ausgezehrten Pollenzellen aber kommen bann in ben Sjonigraum, mohin fie ihrem urfprünglichen 3roecke nach nicht hingehören und für bie herangemachjene $\mathfrak{B r u t}$ in ber unteren Etage gehen jie verloren, ba bekannt= lich ber Pollen nicht umgetragen mirb. Noch bienenwibriger ijt bas Zer= reï̈en des innigen Bandes, das zwifhen auslaufender Brut und eier= legender Rönigin bejteht, welches Durch das Umhängen jomohl bei Preú

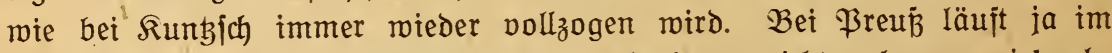
Brutnejt ber abgejteckten Rönigin Brut überhaupt nicht mehr aus, vielmehr werden die bedeckelten $\mathfrak{B r u t w a b e n}$ immer mieder $(6-7 \mathrm{mal})$ in den 'Sonig= raum gebängt. In einem georoneten $\mathfrak{B}$ rutnejt läuit neben ber Rönigin jtets die Brut aus der vorhergehenden Brutperiode aus, jo bá burch bie Brutnejtordmung von felbjt Rönigin und jüngite Bienen zujammenkommen. 2uf biejer Iatjache beruht aber auch folgerichtig bie andere, bá an állen Punkten im Brutnejt $\mathfrak{B}$ edürinis und $\mathfrak{B}$ efriedigung, b. h. jüngere $\mathfrak{B r u t m a d e n}$ und jüngere $\mathfrak{B r u t b i e n e n , ~ a ̈ l t e r e ~} \mathfrak{M a d e n}$ und ältere $\mathfrak{B r u t b i e n e n ~ u j w . ~ z u j a m m e n = ~}$ kommen, dá̉ die dem Brutnejt entwachjenen Bienen als Baubienen an rechter Stelle erjheinen und jo bie Berjafiung und Drganifation bes ganzen Biens an allen Punkten gemahrt und bejchügt merden. 2luf dieje höchit michtigen biologifchen Dronungen im Bien nehmen die lumbänge = und 2lbjteckimker überhaupt keine Rückijcht, obgleich boch gerabe auf biejen bic gebeihliche Entwicklung, die georonete Ernährung und bas ganze Gedeihen des Bienenvolkes beruhen. - Wenn von \$reub und anderen bahingegen an=

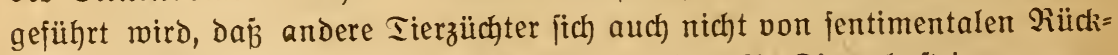
fichten auf ihre Zuchtobjekte leiten lafien und 3 . $\mathfrak{B}$. Tiere kajtrieren, wenn es gillt, ein bejonderes Zuchtziel zu erreichen, fo ermidern wir, daf es bei uns keineswegs Gentimentalität iit, was unts zum $\mathfrak{W i d e r i p r u c h ~ n o ̈ t i g t , ~}$ jondern die offenkundige Tatfache, Dáf es möglich ift, die uns bciden in gleicher Weije vorichwebendem Ziele auf befiere, naturgemäbere Wcife зи erreichen. Das gilt es nun zu berweifen.

Wir haben vor etwa 30 Jahren fchou Den übergang von ber $\mathfrak{B} e=$ handlung von hinten żu ber von oben vollzogen, zunächit unter Beibchaltung 
Der 9lormalganzrabmen im $B$ rutnejt, die wir aber auj 17 vermehrten. Wir jahen uns bamals auch ebenjo wie Preub als Frühtrachtimker ge= zwongen, durch $\mathfrak{B r u t n e j t e r w e i t e r u n g ~ d e n ~} \mathfrak{B i e n}$ auf bie $\mathfrak{S}$ öhe zu führen und ihn zugleich möglichjt vor unzeitigem Schmärmen zu bemahren. Wir jind damals etrạs anders vorgegangen: Wir haben jtets zunächjt vom Zentrum Des $\mathfrak{B r u t n e j t e s}$ aus, jobald bort bie brei Wochen vorher gelegten (Eier als reife, Bienen ausjchlüpften und die Rönigin wieder mit ihrer Eierlage ins Zentrum des Brutnejtes zurïckkehrte, leere, ausgebaute, tadelloje Brut= waben als Ermeiterungswaben eingefügt, damit der Rönigin an erwünjchter Stelle Zellen zur 2lblage ihrer reifenden Eier zur $\mathfrak{B e r j u ̈ g u n g ~ g e f t e l l t , ~ D a s ~}$ Brutneft zugleich vor Unoromung berwahrt und bie Brut wejentlich per= mehrt würde. Sobald Bruttrieb auftrat, ließ̉en wir am Brutnejt künjtliche 2littelmände ausbauen und hingen bieje bann jtets ba ins Brujtnejt ein, mo jich die Rönigin mit ihrer Eierlage befand, erweiterten bamit fort= laufend bas Brutnejt, ohne jeine Dronung zu ftören, ja umgekehrt, indem wir burch bie regelrechte. Errmeiterung die fonjt bebrohte Dronung fchübten. Uuf bieje weije murbe auch bas Brutnejt fajt bis zur Själfte afljährlich erneuert, der $\mathfrak{B a u}=$ und 2 rbeiterbruttrieb in erjchöpfender Weije in 21ftipruch genommen und Brutnejter bis 15 Waben llmfang erzielt.

Bei Behandlung won oben machte dieje miederholte Ermeiterung keine Echmierigkeit, da ja niemals eine Wabe aus ber Wohnung herausge= nommen zu werben brauchte, jondern in bequemiter Weije Runjtraben wie Ermeiterungswaben an richtiger Stelle won oben her eingeichoben werden konnten. Selbit bei einem Stande von etwa 100 Bölkern war Dieje 2 rbeit in kürzejter Zeit vollzogen. Waren bie $\mathfrak{V}$ ölker auf bieje Weije planmäß̉ig und unter voller Berückjichtigung Der Entwicklungsgejebe bes Biens bis zur Sfaupttracht auf die S̈öhe Der Flugkraft gebracht, Dann murbe ein halbhoher Sjonigraum mit 14 Rahmen aufgejebt und, menn jonjt fich bie Tracht= und Witterungsverhältnifje günjtig gejtalteten, wurbe berielbe in kurzer Zeit gefüllt, áusgejchleudert und wieder aufgejebt. Bei diejer Behandlung erfolgten bie Schroärme felten vor ober mährend der Sauptracht, da ja die Triebkraft bes $\mathfrak{B o l k e s}$ ftets jo ausgenübt murde,

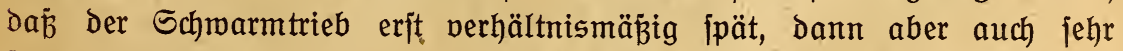
jtark auftrat.

Wer die $\mathfrak{B}$ b̈lker vor dem 2lusjchwärmen bewahren wollte, konnte felbjtoerjtändlich auch Durch Einjtellen eines 2rbjperrgitterichîdes an ge= eigneter Stelle bies leicht und bequem erreichen. Wir haben bies Mittel jedoch bamals nicht angerwand, weil wir ohne dies Zुmangsmittel unjer Biel erreichten.

Gerabe bieje auf 15 Brutwaben ermeiterten Brutnejter auf Normal= ganzrahmen haben uns mun erkennen Iaffen, dá̉ das Normalmá̉ jür die Entwicklung eines gejunden und ftarken $\mathfrak{B}$ olkes viel zu eng war und dá̉ 

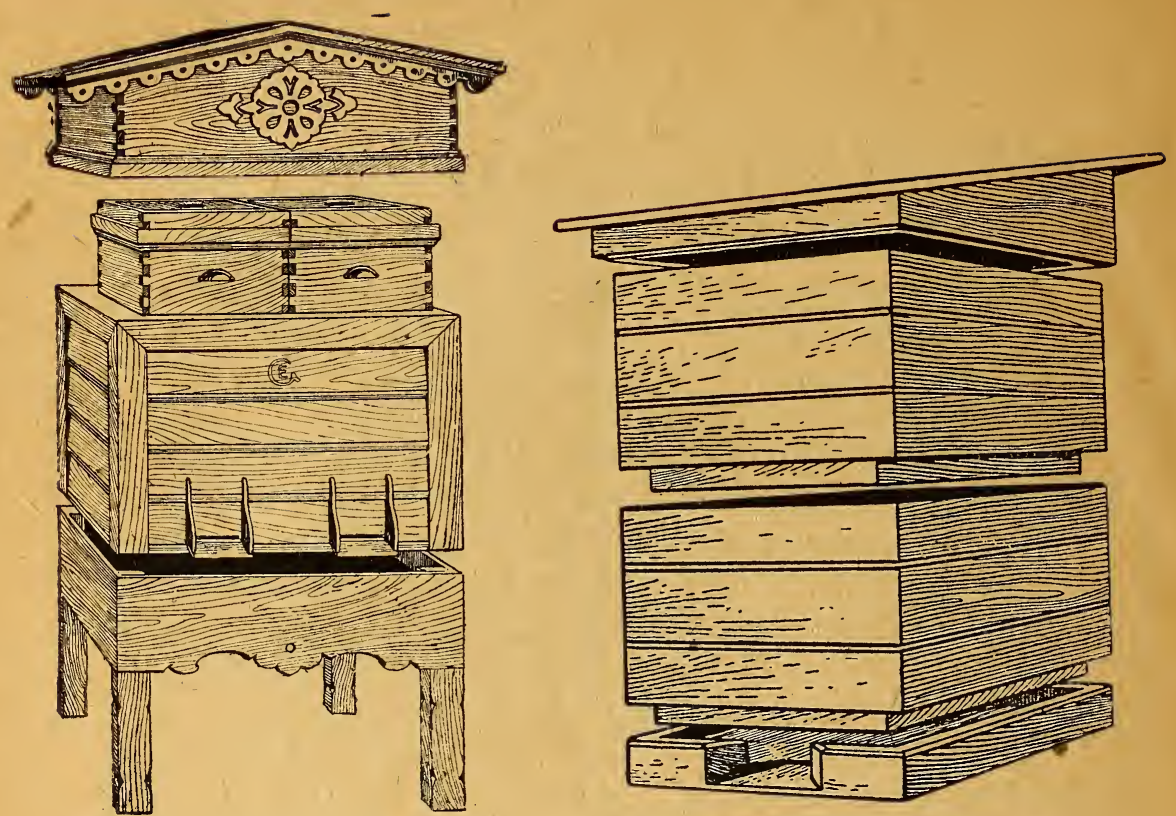

Thüringer 3milling mit. Sodkel und Dach.

; Thüringer Lagerbeute mit berweglichem Bodenbrett.

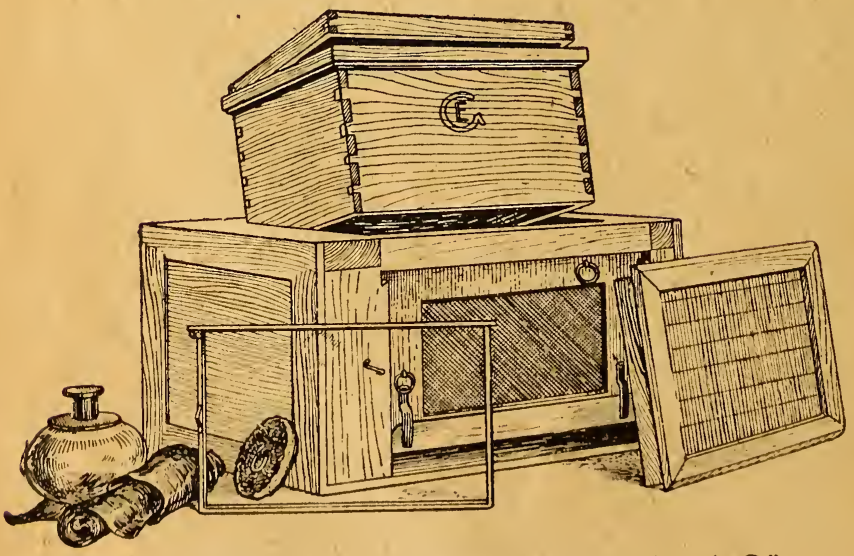

Thïringer Lagerbente mit 2 Binkelnij(henfluglo(h) und Tür.

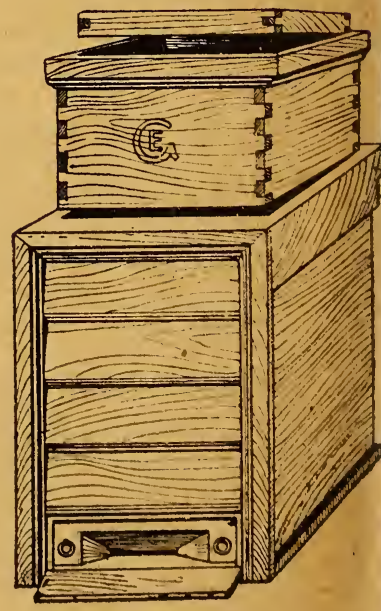

Thürituger Einbeute mit allicitiger früllutg. 


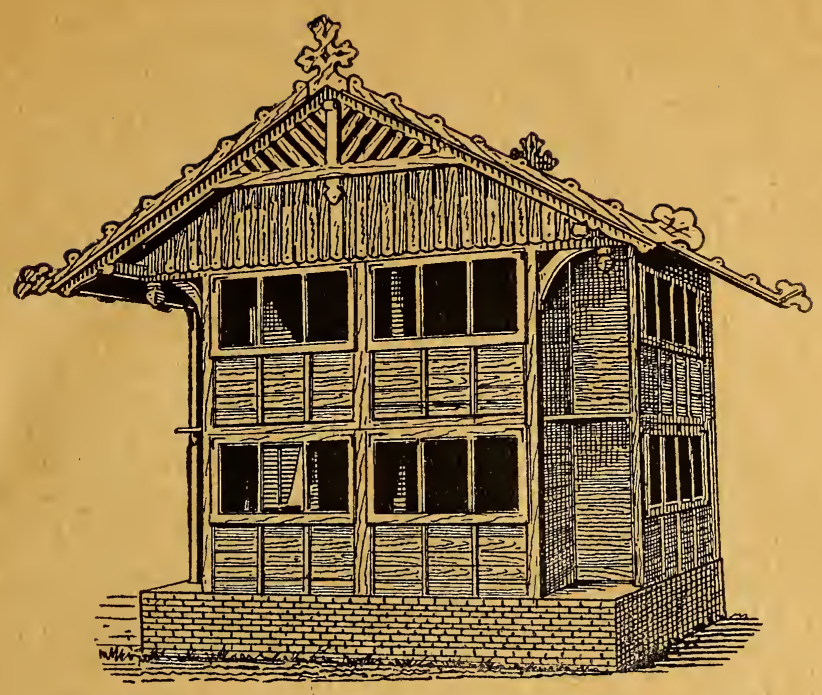

28 fächeriger $\mathfrak{B a v i l l o n ~ m i t ~ T h i t r i n g e r ~ S t a ̈ n d e r b e u t e n t . ~}$

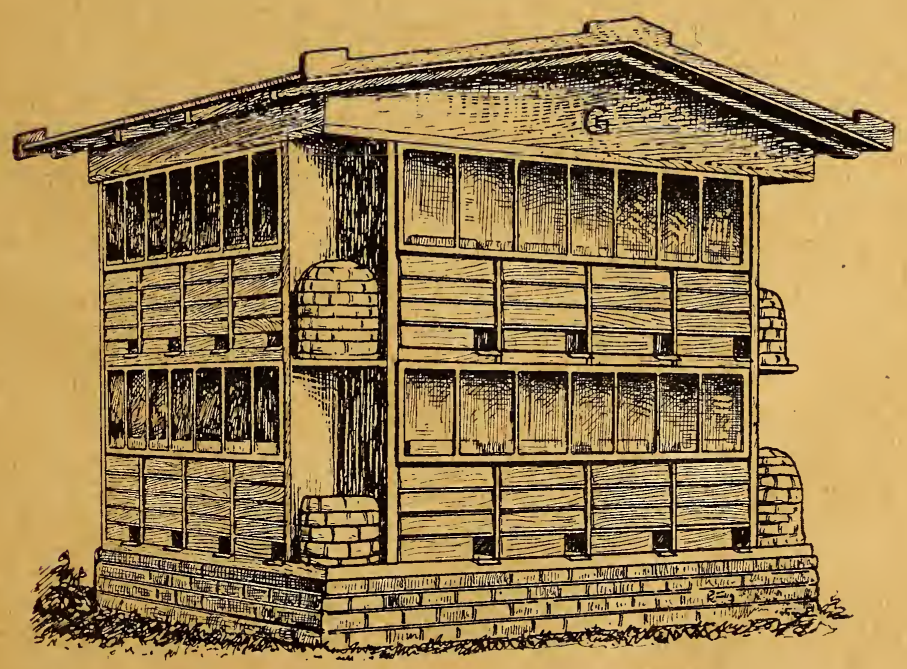

Bienenhaus für 24 \&agerbeuten. 
die immer wieber jich notmendig machende Ermeiterung zumeijt nur eine Folge der Bienenmibrigkeit des Normalmaß̉es mar. Deshalb jind wir bann nach und nach zu bem Durch umfangreiche praktijche theoretijche Berechnungen fich als richtig und zweckmäßig erwiejenen jo= genannten rationellen $\mathfrak{M a} \dot{B}$ von $40 \times 25$ qcm wachsfläche über gegangen, bei welchem neun Waben Das eigentliche $\mathfrak{B r u t n e j t ~ b i l b e n , ~ w a ̈ h r e n d ~}$ die Bruträume für zrölf Rahmen 2iaum boten, einmal, damit die Beute Spielraum zur Behandung bot, ohne dá̉ man je eine wabe aus der Wohnung zu entnehmen brauchte - mas für die Behandung des Biens von ganz auß̈erorbentlicher Bebeutung und von größztem Borteil ijt Dann auch, um Raum für etroa notwendig ericheinende Ermeiterung des Brutnejtes und für bas Uusbautenlajfen von Runjtraben zur Berfügung zu haben. Damit war ein gewaltiger Fortjchrtt vollzogen worben, der zu einer naturgemäß̈en, den Sebens= und Entwicklungsgejeben voll ent= iprechenden Bienenpilege geführt hat und zugleich die ganze Bienenzucht= praris auf eine neue, beffere Grundlage geftellt hat, Deren heilvoller cin= flü fich von Jahr zu Jahr in fteigendem Mẳe in ber ganzen Jmkermelt geltend gemacht und eine völlige Reform unjerer ganzen vaterländijchen Bienenzucht zujtande gebracht hat. Dazu ījt die jogenannte Ihüringer Bienenwohnung jowohl in ber Ständer = wie Sagerbeutenform jo einjach und jo anpaijungsfähig an alle erbenklichen Betriebsverhältnijie, da ja fie in diefer Sjinjicht ohne überhebung wohl als unübertrefflich bezeichnet werben kann.

Wir jegen die Bekanntjchaft der verehrlichen Sejer mit diejer in allen Gegenden verbreiteten Stockform voraus, jo baß̈ wir uns eine ausführltche Bejchreibung berjelben erjparen können, da es uns hier ja nur auf die Darjtellung Der Betriebsmeije in Frühtrachtgegenden in unjeren Beuten an= kommt. Wer aber die verjchiedenen Uusführungen der Thüringer Bienen= mohnungen noch nicht kennen jollte, den verweijen wir auf unjer Sehrbuch "Der Bien und feine $\widetilde{3}$ ucht", auch bitten wir iijn, fich von ber "Deutjchen Bienenzuchtzentrale" in DB̉mannjtedt $i$. Th. einen Ratalog kommen zu lafjen, in melchem alle Stockformen im Bilde vorgeführt und beichrieben find.*) Die Ubbildungen der bemährteiten Formen haben wir Geite 16 und 17 zum 2lboruck bringen lafjen.

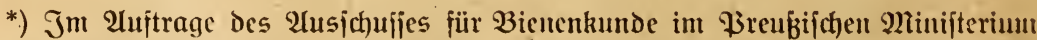

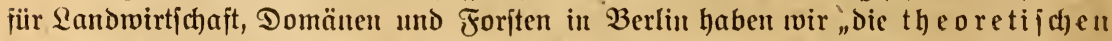

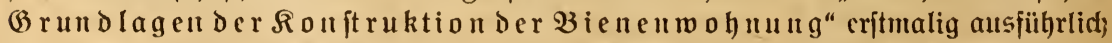

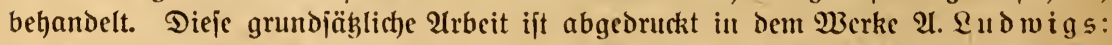
"Llnjere $\mathfrak{B}$ ienen" II. Teil. (Verlag vou Frib Pjenningitorff, Berlin W 57.) W3er jid) ein jachkundiges Urteil über Die j(hwierigite aber aud wichtigite Frage Der

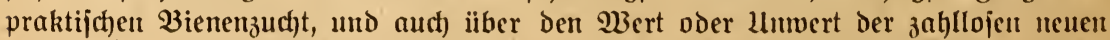
Bienenwohnungskonftruktionen biloen will, muß dicje Ubhandung gelejen haben. 
Die Thüringer Bienenwohmung bietet jomohl in ber Ständer= wie auch in ber \&agerform bem Bien in bem Brutnejt von, neun $\mathfrak{W a b e n}$ von $40 \times 25 \mathrm{qcm}=1000 \mathrm{qcm}$ Wächsfläche eine $\mathfrak{B r u t k u g e l}$ von $25 \mathrm{~cm}$ Durchmefier in Sjöhe, Breite und Tiefe Dar, welche 35000 Brutzellen umfät. (Beht Das Brutnejt bei aufjeteigender (Entwicklung, wie bies tatjächlich regelmäß̈̈ig gejchieht, von ber Rugelform in bie Eiform ïber, fo finden fogar 50000 Brutzellen Unterkunjt, aljo jo viele, als eine gute Rönigin burchichnittlich auf Dem Sjöhepunkte Der Entwicklung vor Dem Schwärmen innerhalb einer Brïtezeit von Drei Wochen mit (Eiern belegt. 2uch bietet ber Brutraum Dann noch Raum zur 2rblage- Der. Pollen= und Sontgzellen an biengemäß̄er Stelle, D. h. Da, wo fie nach Der inneren Drganifation bes Biens hin= gehören, was von ausjchlaggebender Bedeutung für bie Ernährung Der Brut ijt. Da bieje Brutnejter ben Entwicklungsgejegen und Bedürfnifien Des Biens bis zum lebten Schmarmbrutją völlig entiprechen, èntfällt ganz von jelbjt bie in bienenmibrigen Wohnungen fich aufbrängende Notwendig= keit Der Ermeiterung bes Brutnejtes fhon im Mlai. Der Bien kann fith ia in biejen Brutnejtern nach feiner anerichaffenen Dronung felbjt ungejtört und ungehindert entwickeln. Nur bann, wenn eine überreiche $\mathfrak{P}$ ollen $=$ und S) onigfrühtracht, z. B. aus Raps, \&öwenzahn, Dbjtblüten und Dergleichen bas bis bahin noch nicht allzu umfangreiche Brutnejt einzulchließjen und bamit an ber ermünjchten, orbmungsgemäßen 2usdehnung zu hindern broht, mús der Smker helfend eingreifen Durch Darbieten einer leeren Brutwabe im Zentrum des Brutnejtes, jobald dort ausjchlüpfende Zellen zu finden

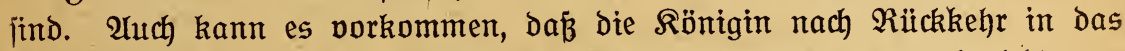
Brutneitzentrum bei jtark anjchellender (Eierproduktion an noch nicht aus= gelaufene Brutzellen ankommt und fich bann gezmungen fieht, dieje zu üherjhreiten, um bie überichüffitgen Eier an Der Beripherie abzulegen. Sobald ber Bienenvater bies merkt, kann er ebenfalls burch Einftellen einer leeren Brutwabe an orbnungsgemäßer Stelle ber Rönigin Zellen an dem für fie ermünjchten Plabe Darbieten, Damit zugleich der reifen Brut Zeit zum Uusjhlïpfen verichaffen und bas ganze Brutnejt vor Unoronung bemahren.

Das Ulles geht infolge Der Behandlung von oben bei unjeren Bienen= wohnungen ipielend leicht und ichnell vonjtatten. In fünf Minuten ijt ein Brutnejt unterjucht und im Bedarfsfalle an rechter Stelle ermeitert, während folche $\mathfrak{B e r r i c h t u n g e n ~ b e t ~ B e h a n d l u n g ~ v o n ~ h i n t e n ~ a ̈ u b e r i ́ t ~ m u ̈ h = ~}$ jam, zeitraubend, ftörend und jafwierig find. Man Ieje z. B. eimmal eine Urmweijung Durch, melche Đreú für Das Umbängen des Brutneites gibt und. man wird fich fofort jagen, daßj fich ein Durchjchnittsimker nur im äuß̉erjten Notfalle zu einer jolchen umitändlichen und jchmierigen Dperation

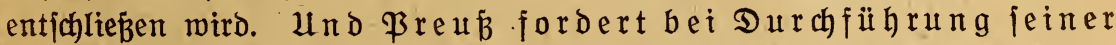
Betriebsweife eine minbejtens jechs=bis jiebenmalige 
Derholung einer ähnlichen Dperation. (Wabenwechjel hinter bem Brutraumichied!) Das bürfte wohl auch die Urjache fein, báp fich bas an und für fich jo gründlich und vortrefflich durchgedactste $\mathfrak{B e r f a h r e n ~ i n ~ D e r ~}$ Imkerwelt nicht burchzufegen und einzuführen vermochte und auch in Jukunft keine weite $\mathfrak{B}$ erbreitung finden wirb.

(Ebenjo leicht und bequem vollzieht fich Das 2usbauenlafien Der Runit= waben und bamit bie weitgehendite Jnanjpruchnahme bes Bautriebes und auf $\mathfrak{B u n i c h ~ a u c h ~ b i e ~ E r n e u e r u n g ~ b e s ~ B r u t n e j t e s . ~ M a n ~ h a ̈ n g t ~ d i e ~ R u n j t w a b e ~}$ an bie biengemäß̉e Baujtelle hinter bie bas Brutnejt abjchließjende Pollen= wabe ein, wo fich jtets von jelbit bie Baubienen anjammeln und bie nötige Wärme herricht. Sit eine $\mathfrak{W a b e}$ ausgebaut und foll bas Brutnejt pon innen her erteuert werben, fo wirb fie jtets ba eingehängt, wo auf ben Naçbar= waben fich auslaufende Brut oder ganz frifche (Eier im Zentrum befinden. Uuf Dieje Weije fügen fich bie neuen Waben oronungsgemäß in Das Brutnejt ein. Die alten $\mathfrak{W a b e n ~ g e l a n g e n ~ v o n ~ j e l b i t ~ n a c h ~ r u ̈ c k w a ̈ r t s ~ u n d ~ k o ̈ n t e n ~}$ jchließ̧lich aus Dem Brutnejt entfernt merden.

2uch die übrigen pflegerijchen Tätigkeiten während biejer aufiteigenden Entwicklungsperiode Iajien fich fpielend leicht in unjerer Bienentwohnung vollziehen, jo z. B. Das Entbeckeln Der alten Sjonigzellen, Das Tränken und bie fpekulative Fütterung fowie etwaige Notfütterung, ba bies ja auch burch ben "Thüringer \&uftballon" von oben her burch bas vorhandente Futterloch in einzigartig leichter und bequemer weipe gejchieht.

2uch wir wenden gern ba, wo wir keine Runjtwaben mebr ausbauen laifen, ben jogenanten Baurahmen,als Triebbarometer als lebte Wabe unmittelbar vor bem Drahtgazefeniter an.

Um liebjten überlajien wir Den Bien, falls fein Brutnejt jonjt in Dronung ijt und er jelbjt in rechter $\mathfrak{B e r f a j j u n g ~ f i c h ~ b e f i n d e t , ~ b a z u ~ a u c h ~ b i e ~}$ Rönigin voll leijtungsfähig ijt, jolange als möglich jich jelbjt und greifen nur da helfend und unteritübend, fördernd und antreibend ein, wo es ñötig erjcheint. Das biengemäß乃e Brutnejt jorgt jđjon von jelbit Dafür, baß̉ bie Entroicklung nach Wunjch flott und ficher vonitatten geht. Whit ben jonjt empiohlenen Eingriffen haben wir zumeijt nichts zu tun und vor Dem Schwärmen brauchen wir im Maai niemals 2ngit łn haben, Da Der Bien ficher bis zum Beginn Der Sfaupttracht Raum genug hat, fich bis zum Söhepunkt zu entroickeln. - Wir find froh, wenn bic $\mathfrak{b}$ ölker unmittelbar vor Der Şaupttracht bie höchjte Flugkraft erreichent, was leider in ungünjtigen Jahren nicht immer ber Fall ift.

Werken wir an Dem Stocken Des Bautriebes beim 2usbauen Der Rumitwaben oder an Dem Baurahmen, dá̧ Der Bient vom Schroarmtricb erfaßit ijt, jo wenden wir als Schmarntverhütungsmittel ben 2ustaujh alss: laufender Brutwaben gegen offene Brut aus f̧wächeren $\mathfrak{B}$ ölkern an, wo= 
burch zugleich eine (Bleichmachung Der $\mathfrak{B}$ ölker vor Beginn ber SJaupttracht fich vollzieht. Sit die ganze Entroicklung normal und güntig verlaujen, jo kommen jtarke $\mathfrak{B}$ ölker in richtiger $\mathfrak{B e r f a f f u n g}$ in bie Şaupttracht, b. h. jolche, in benen bie Flugbienen jehr zahlreich find und im günjtigen Ber= hältnis zu den Şausbienen und der Brut jtehen.

Gerade biejer Bunkt wird jelten genug berückjichtigt. Die Errahrung lehrt, daß̉ nicht alle bem 2ugenjcheine nach gleichitarken $\mathfrak{B}$ ölker, melche auch gleichbiel Bienen in bie gleichreiche Tracht jenden, gleichgroß̈e 5 jonig= erträge lieferm. Dft jteht ba ber Smker vor einem Rätjel, Das er nicht zu löjen vermag. Zumeijt ijt Der Grund für dieje Berjchiedenheit bes Fjonig= ertrags gleichjtarker $\mathfrak{B}$ ölker barin zu juchen, Daßj in Den ausjchlaggebenden Trachttagen - und oft find bas nur 8-14 Tage - ber Brutjtand in ben $\mathfrak{B}$ b̈lkern verjchieden ift. Das eine $\mathfrak{B}$ olk hat zahlreiche offene Brut= zellen mit gefräß̉igen Maben, ein anderes bagegen verhältnismäß̈ig viel geichloifene $\mathfrak{B r u t}$ bei geringer Zahl offener $\mathfrak{B}$ rutzellen. (Es ijt jelbitwerjtändlich dá̉ Das eritere $\mathfrak{B}$ olk an Sonigertrag hinter bem lebteren zurückbleibt, da Der Eigenbedarf Defielben an SJonig bedeutend größ̈er ift, wie bei Dem anneren. - Nun lehrt aber auch die Errfahrung, dá̉ eine einjebende itarke Sonigtracht nicht nur gleichjam mechanijch ben Brutanjag, aljo auch bie offene Brut, einjchränkt; indem alle auslaufenden Brutzellen mit Sonig gefüllt werben, jondern auch auj phyjtologijcher Grumblage zu einer $\mathfrak{B} e r=$ minderung ber $\mathfrak{B r u t e n t f a l t u n g}$ hinführt, ba eine einjeitige itarke Sjonig= tracht bie (Eierlage eher vermindert als erhöht. Daraus erklärt fich ja auch die Richtigkeit Des alten Erfahrungsjäes, Dá̉ reiche Syonigjahre ichlechte Echrarmahre find und umgekehrt: Jahre mit reicher Bollentracht bei verhältnismäß̈ig geringer Sontgtracht ;ausgejprochene Schnarmjahre jint.

Da mit Beginn Der Şaupttracht Der verhältnismäß̈ig groß̉e Sonig= raum aufgejebt wirb, io hilft auch bas bann wejenilich mit, ben etwa vorhandenen Schwarmtrieb noch einmal etwas zurückzudämmen, ba ia die Bienen zunächit noch 2 rbeit bie SJülle und Fülle haben. Recht umer= wünjcht ijt es uns freilich auch, wenn kurz vor ober während ber Sfaupt= tracht ein Schwarm fällt. Gejchieht bies boch wiber unjeren Willen, jo werfen wir Denjelben auf ausgebaute Waben, damit er mit dem den Schwärmen eigenen (Eifer boch bie Sjonigtracht für uns ausnüben mús. 2uf bieje weije geht uns bon ber Sjonigern'e nichts verloren. Unter Umitänden wird auch Der Schwarm an bie Stelle bes Wutteritockes ge= jegt und ber Mutterjtock an eine neue Stelle, um das Nachjchrärmen zu verhüten und Dem Schwarm alle Flugbienten des Mutteritockes zuzuführen.

Wer nun aber während Der Saupttracht auch in jchmarmreifen $\mathfrak{B} \not ̈ l=$ kern Das Schwärmen jicher verhüten will, ber kann ja auf benkbar ein= fache Weije bie Rönigin Durch ein jenkrechtes 2bjperrgitterichied, welches wieder von, oben her bequem und mühelos an britter oder vierter Stelle 
von vorn eingejebt mirb, auj den hinteren Teil des Brutraumes bejchränken unter Einfügen eines 2 biperrgitters auch zwijchen Brut= und Sjonigraum. Eine meitgehende $\mathfrak{B}$ ejchränkung ber (Eierlage braucht bamit nicht verbunden zu jein, zumal da in biejer Zeit bie reiche Tracht fchon bas Brutnejt hie und ba von jelbjt einengt. Wir menden auch biejes Mittel mur in aus= geiprochenten Schmarmjahren an, ba wir unter normalen $\mathfrak{B e r h a ̈ l t n i f j e n ~ n i c h t ~}$ unter übermäßjiger Schmarmlujt jelbjt ftarker $\mathfrak{B}$ ölker zu leiben haben. Wenn bann am Ende der Tracht, in hiefiger Gegend zumeift vom 20. Juni $\mathfrak{a b}$, Schmärme fallen, fo find uns biejelben millkommen, ba fie uns jungen Bau jchaffen, bas Material barbieten zum Uusfüllen von Sücken auf bem

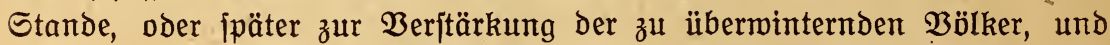
da bie abgejchmärmten $\mathfrak{V}$ b̈lker burch ben Echmarmakt bie Rönigin ver= jüngen und ben $\mathfrak{B a u}$ einmal gründlich jäubern.

Damit kommen wir aber auf bie legte Entjcheibungsfrage: Jit mit

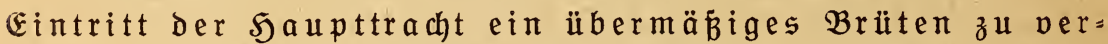
hüten? Sit alfo ein 2rbjtecken ober Ubjperren ber Rönigin auf brei, zwei oder gar eine $\mathfrak{W a b e}$, wie es von Preuß́ und vielen anderen als unbedingt nötig empfohlen, ja gefordert wird, wirklich ratjam, ober läp̧t fich Derjelbe Zneck nicht auf einfachere und naturgemäpere Weife erreichen? Das ift ficherlich bie Frage, welche heute im $\mathfrak{V}$ ordergrumbe bes Interefies Der Imkermelt jteht und bei melcher bie 2rnjichten und Wege weit aus: einandergehen. Wir glauben nicht fehlzugreifen in ber Ưnahme, baß̉,

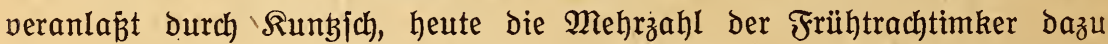
neigt, Den Ertrag an Sjonig jich fajt ausjchließjlich von ber Brutbejchränkung abhängig zu benken, jo Daßj heute in meiten Rreijen als oberjter (Brund: fab ber Bienenzucht in Frühtrachtgegenden ber gilt: Ohne weitgehen bite $\mathfrak{B r u t b e f c h a ̈ n k u n g ~ i n ~ b e r ~ S H a u p t t r a c h t ~ k e i n e ~ b e f r i e b i g e n d e ~}$

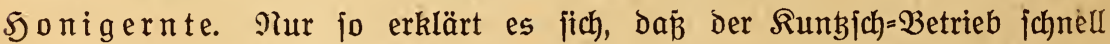
viele Unhänger gefunden hat, von benen freilich bald auch mieber eine groß̉e Zahl fahnenflüchtig gemorden ijt, ba trob aller llmjtändlichkeit ber Behandlungsweije Die Erfolge den Ermartungen keinesmegs entiprochen

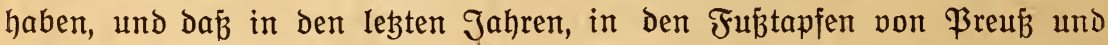
Runbjich mandelnd, eine ganze Rieihe Bienenmohnungen "erfunden" morben find, welche in ber Sjauptjache barauf angelegt find, bie Brutbejchränkung

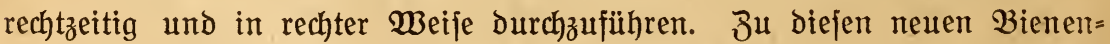
wohnungsformen, welche in Der "Brutbejchränkung Das Sheil Der Bienen= zucht juchen, gehören, foweit wir jehen können, außer Dem Preub'jichent

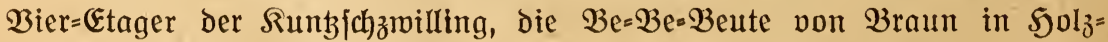
haujen, Der "Fुonigquell=Stock" pon Rraufe=SJarttung itt Frankfurt a. D. D.,

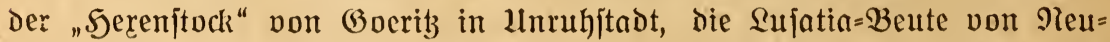
mann=Rraufe in WBaldorf $i$. S., auch bie Rhanbeute $u$. a. m. Der ver=

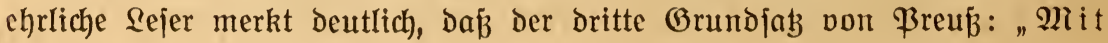


Eintrittund während der SGapttrachtzeit iftein übermäßiges $\mathfrak{B r u ̈ t e n ~ b e r ~} \mathfrak{B}$ ölker zu verhüten " in Deutichland Schule gemacht hat; bie alte Dzierzoniche "Diamantene Regel", Die man ichon als einen für immer überwumbenen Standpunkt hat erklären mollen, feiert in jeder neuen Beuteform eine neue 2uferitehung, jo dá̉ es uns

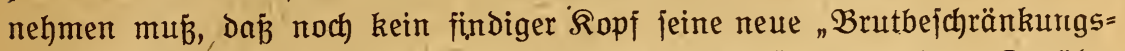
beute" Die $\mathfrak{B}$ eute der "biamantenen Regel " getauft hat. Darüber hinaus gäbe es boch bann nichts mehr an zugkräftigen Namen zu erfinden, auf welche Unfänger fo Ieicht hineinfallen, wenn nur bie entjprechende Reklame Dafür gemacht mirb.

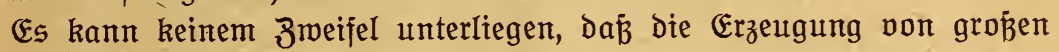
Mengen 'Bienenfleijch für jicher trachtloje Zeiten, in benen bies unnübe Bienenfleifch nur ben von früheren (Bejchlechtern für ben Winter und auch für Den Smker eingejammelten Sjonig wegfrift und aufzehrt, ein zweck= wioriges $\mathfrak{B}$ eginnen barjellt. Nun find eben alle ausgeiprochenen Frühtrachtgegenden jolche, in Denen mit Dem 2lnjang Der Getreideernte tat= jächlich jegliche nennenswerte Tracht aufhört, $\delta a \tilde{B}$, aljo jpätejtens der 1. Uuguft, hier und da jogar ichon ein früherer Termin als Trachtichlús ju betrachten ijt. Diefe Tatjache unberückichtigt lafjen bei Iufitellung eines betriebsplanes hiebe fich event. um den ganzen Ertrag aus ber Bienenzucht bringen. Nun liegt ia ber ธchlǘ für jeden denkenden Jmker fehr nahe: War es möglich, für die Saupttracht Durch ipekulative Fütterung und Erweiterung Des Brutnejtes genau fünf Wochen vorher die Flugbienen herworzurufen, jo ift es ebenjo möglich, die Erzeugung von unnübem Bienenfleifch für trachtloje Zeiten DaDurch zu verhüten, daß̉ man die Rönigin einfach fünf Wochen vorher und weiter hinbert, Eier in größ̈erer Menge zu legen. Die $\mathfrak{B r u t b e j c h r a ̈ n = ~}$ kung auf irgend eine Weije muß̈ Demnach jchon ipätejtens Ende Juni einjegen, menn am 1. 2ugujt endgültiger Trachtichluß ijt, auch noch früher, wenn ber Trachtichlǘ früher beginnt. Wie einjt Dr. Dzierzon in Dem Einkapjeln Der Rönigin bie "Diamantene Regel“" entbeckt zu haben glaubte, fo meinen heute alle Frühtrachtimker in Dem Ubjtecken oder Ubjperren Der Rönigin während Der Şaupttracht auf brei, zmei ober gar nur eine Wabe Den Stein ber Weijen gefunden zu haben, und Preub und Runbjich jind es gemejen, welche dieje Betriebsmeife jcharfitnnig ausgebaut und angemandt haben, ja legterer hat fie jogar zur Grundlage für bie Ron= itruktion feiner Bienenwohmung gemacht. Und wer zählt bie Bälker, nennt die Namen, welche nun auf bie Betriebsweife biejer Männer ein= gejchworen find? Wan jagt nicht zu viel, wenn man erklärt, Daß̉ heute bas jogenannte 2 bjteckperfahren neben dem jogenannten Umbängen in ber Deutichen Jmkerwelt $\mathfrak{M}$ o defache geworden ift und ber nicht $\mathfrak{U n}_{\mathfrak{n}}=$ ipruch auf ben Namen eines fortjchrittlichen Imkers erheben barf, welcher 
nicht bie Brutbejđränkung in ber einen ober anderen Form anțendet. 2usfchlaggebend für bie Empfehlung fint aub̄er bem Sinmeis darauf, baß́ unnü̈ge Freffer zur Unzeit verhütet werden, bie Behauptung, ১á̉ bie Sjonig= ernte aus Der Şaupttracht ganz wejentlich Durch Das 2rbjperren ber Rönigin erhöht werde, indem jelbjtverftändlich nicht joviel Brut zu ermähren ift, welche bekantermak̄en jehr viel $\mathfrak{P o l l e n}$ und Sjonig verzehrt und - was jtets, jo auch von Preub́ bejonders hervorgehoben wird - weil Taujende von $B$ ienen für die Sammeltätigkeit frei werden, welche fonjt häuslicher (Bejchäfte, vor allem ber Brutpflege wegen im Stocke bleiben mü̈bten. Dem unkundigen Smker leuchten natürlich jolche, jeinte Selbitjucht befriedigenden Gründe fofort ein und überzeugen ihn bavon, bá̉ bas 2 b= fperren bie unerläßliche Bebingung und $\mathfrak{B}$ orausfebung einer reichen, un= geichmälerten Sontgernte jei und jo ift zur Zeit: 2 b jtecken der $\Re$ önigin während ber SGapttradt $\mathcal{E}$ ofung und Feldgejdrei in ber gejamten Jmkermelt. Da ijt es natürlich richt leicht, gegen ben Strom zu jchmimmen und zu ruhiger überlegung und jachkundiger und bejonnenter 2 brwägung Der Gründe, welche für und gegen bieje Betriebs= weife fprechen, aljo ihrer $\mathfrak{B}$ orteile und Nachteile, aufzurufen utto barauf

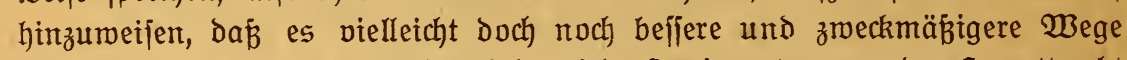
gibt, bas allen vorichwebende Ziel: reiche Sjortigernten aus ber Saupttracht und $\mathfrak{B e r h u ̈ t e n ~ v o n ~ u n n u ̈ b e m ~ B i e n e n f l e i f c h ~ i n ~ t r a c h t i ́ p j e r ~ Z e t t ~ z u ~ e r r e i c h e n . ~}$

Selbitweritändlich hat es bei $\mathfrak{P r u ̈ f u n g}$ diejer Betriebsweije keinen Zెeck, Behauptung gegen Behauptung zu ftellen, wie bas leider nur zu oft bei ber Erörterung Diejer Frage gejchehen ijt, jondern es gilt, offenkundige, unanzweifelbare Tatjachen ins Feld zu führen. Welche Tatjachen von Den 2nnhängern Derjelben geltend gemacht werben, haben wir joeben ichon ge= zeigt. Jebt gilt es, bieje auf ihre Richtigkeit und Stichhaltigkeit zu prüfert.

Es muß unumwunden zugejtanden werden, dá̉ das eitte Ziel: $\mathfrak{B} e r=$ hüten von untübem Bienenfleifch zur Unzeit, Durch das 2lbiperren zur rechten Zeit, aljo etwa fünf Wochen vor Trachtjchluß́, ficher erreicht wirb, - wir bejtreiten aber ganz entichieden, dá̧ damit zugleich eine mejentliche Erhöhung Des Sjonigertrags aus der Sfaupttracht erzielt wird und gebent erit zu überlegen, ob ber Echaden, Der burch Llnterbrücken ober $\mathfrak{B} e=$ jchränken des 3 ruttriebes in jeinen Folgen für bie $\widehat{J}^{u k u n f t}$ nicht viel größjer ijt, als ber freilich in bie Augen fallende momentane Nuben.

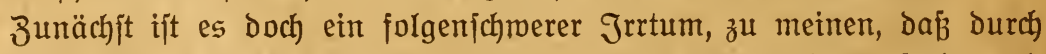
Unterbrücken und Bejchränken ber $\mathfrak{B}$ rut in ber Sjaupttracht taujende und abertaujende SJausbienen frei würden für bie Sammeltätigkeit. Die Biente braucht, vom $\mathfrak{E} \mathfrak{a} \mathfrak{a b}$ gerechnet, wenigftens 36 Iage, vom 2usfichlüpfen $a \mathfrak{b}$ gerechnet 14-15 Tage, ehe fie Flugbiente wird, Damit ift fie aber noch keineswegs auch Trachtbiente. Wir nehnten auf (3rumb von Beobachtungen vielmehr an, dä̉ fie Durchichnittlich erjt am 19. Tag wirklich Irachtbiene 
miro und noch etwas fpäter erit im bejonderen Sinne Sjonigiammlerin. Das find biologifhe Tatjachen, die unumitöblich fejtitehen. Deshalb fliegt aber auch keine junge Biene etwa eine Etunde früher auf Tracht aus, nur beshalb, weil fie wegen der Brutbejchränkung im Etocke nichts zu tun findet. $\mathfrak{B i e l m e h r}$ hocken bann bieje jungen Bienenjcharen untätig und träge im Stocke herum, ober, wie es bie Tatjachen lehren, liegen, zumal bei groß̉er Sjibe, in ungeheuren Mengen vor Dem Stocke, obgleich bie reichjte Tracht zum 2lusflug lockt, und bieje jungen Bienen= icharen, melche keine Befriedigung ihrer Triebe im Mutteritocke mehr finden, find vor allem die "Echmarmteufel“, ঠ. h. Die Erreger des Echmarmtriebes! Man beobachte boch einmal einen Fegling, in welchem fich ausfchlieblich junge Bienen befinden. Dem Fegling fehlen boch zunächjt alle älteren - Flugbienen und wenn es möglich wäre, würden boch bie jungen Bienen ausfliegen, um die dringenden Bedürfniffe des Etockes zu befriedigen, aber es fliegt zunächjt und längere Zeit auch nicht eine Biene aus, nach einigen Tagen erfolgt das erite fahwache $\mathfrak{B}$ orjpiel, bann ericheinen wiederum ipäter $\mathfrak{P o l l e n j a m m l e r i n n e n ~ u n d ~ z u l e g t ~ a u c h ~ S o n i g i a m m l e r i n n e n . ~}$

Damit ift boch burch Das Berhalten ber Bienen unzmeideutig nach= gemiejen, Daß̉ junge Bienen unter keinen Umitänden jtellvertretend bie Tätigkeiten älterer Bienen übernehmen und erfüllen können. Damit fallen aber auch alle Spekulationen auf Erhöhung ber

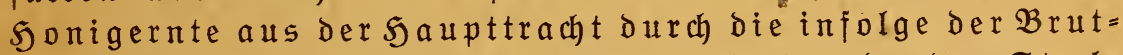
befchänkung für bie Sammeltätigkeit freimerbenden 5 tock= bienen haltios in jich jelbjt zujammen! Uber dieje grumberkehrte Meimung ift einer von den Jrrtümern, die unausrottbar in ben Imker= köpfen feftitgen und immer wieder ihre verberblichen Wirkungen auf die Betriebsmeife der Bienenzucht, ja jogar auf bie Ronftruktion ber Bienen= mohnung ausüben!

Ja, bie Eache hat noch einen ganz bejonderen und bedenklichen Saken! Die Erfahrung lehrt mit wünjchenswerter Deutlichkeit, Dá̉ Bienen, melche ihr Triebleben im Sjerbite nicht mehr ausleben können, Den Winter überdauern, ihr Triebleben in fajt ungefchmächter Rraft ins kommende Jahr hinüberbringen und acht bis neun Monate alt werden. Dagegen iteht ebenjo feit, dá̉ während der flottejten Entwicklung des Biens die $\mathfrak{B i e n e n ~ m i t u n t e r ~ n u r ~ j e c h s ~ b i s ~ a c h t ~} \mathfrak{W}$ ochen alt werden, was bemeift, dáz das Triebleben um fo ichneller verbraucht wird und erlöicht, je intenfiner es fich betätigt. OUus biejer unleugbaren Tatfache ergibt jich aber folgerichtig, daß́ bie Bienen, welche infolge ber Brutbejchränkung ihr

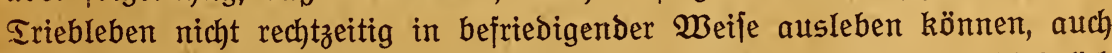
erit ipäter in bie nachfolgenden Triebformen übergehen, alfo wahricheinlich fich nicht etwa früher, fondern vielmehr erft fpäter aus Brutbienen zu Flug= bienen entwickeln. Wenn bas auch noch nicht erakt bewiejen worben ift, 
fo liegt Doch bie Wahricheinlichkeit jehr nahe und zeigt, Daßj ber erhoffte Erjolg Der Brutbefchränkung, welcher in ber EErhöhung Der Sjonigernte Durch vermehrte, freimerbende 2rrbeitskräfte bejtehen foll, in Wirklichkeit nicht eintritt thnd nach Sage aller $\mathfrak{B e r h a ̈ l t n i f i e , ~ w e l c h e ~ v o n ~ a u s j h l a g g e b e n t e r ~}$ Bedeutung für dieje Frage find, nicht eintreten kann. Wir hoffen, Daß nunmehr biejer Grund nicht mehr und nicht wieder für bie Sjeiljamkeit ber Brutbejchränkung in ber SJaupttracht angeführt merben wirb.

Weiterhin ijt aber auch wohl zu bedenken, was mir ichon oben kurz erwähnt haben, daß̉ jede Bruteinjchränkung eine Sjerabjebung bes Eifers Des Biens in feiner Sammeltätigkeit zur Folge hat. Dieje Tatjache, dá̉ mit Der $\mathfrak{B r u t}$ ber Flei $\mathfrak{3}$ Der $\mathfrak{B}$ ölker in gerabem $\mathfrak{B}$ erhältnis jteht, ijt jo

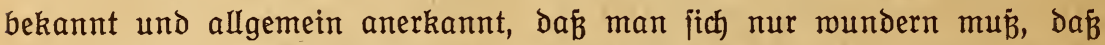
fie gerabe bei ber Mobejache ber Brutbejchränkung nicht beachtet mirb. Zeigen boch ichon die $\mathfrak{B}$ ölker, welche ben jungen Bienen nicht mehr genügend Befriebigung ihrer Brutgier in Gejtalt von gefräpigen Maden verichaffen können, einen auffälligen Rückgang ihres Sammeleifers und ein jtarkes 2Injhmellen Des Schmarmtriebes, was bann erit recht eine Err= Iahmung des Sammeltriebes zur naturgemäßen Folge hat, während alle Völker, welche noch in flotter Brutentwicklung jtehen, auch ben höchjten Sammeleifer entfalten. Schon biefe offenkundige Tatjache ift für uns bis= her bejtimmend gemejen, Die Brutbejchränkung oder gar Brutunterbrïckung während Der SJaupttracht nicht anzumenden, ba wir von innen keine Er=

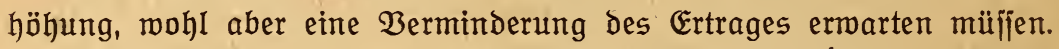

Ebenjo haben wir fhon wiederholt Darauf hingemiejen, bá Durch die meitgehende Brutbejchränkung höchit bedenkliche \&ücken in ber $\mathfrak{V}$ olks= gliederung bes Biens entjtehen, welche ihren verderblichen Einflúb jowohl bei Der jpekulatinen Sjerbitfütterung, wie unter Umitänden erjt recht bei ber überminterung und ber Frühjahrsentwicklung im folgenden Jahre geltend machen. Wir brauchen bieje Tatjache nicht nochmals näher zu begründen, da fie ja jedem kunbigen und icharfitchtigen Imker fojort einleuchten mú, und eigentlich in ihrer bedenklichen Tragmeite von niemand bejtritten merben kann.

Wir haben Daher jømere Bedenken, nur um bes kurzjithtigen Zieles willen, Bienenfleifch in trachtlojer Jeit zu verhüten, jowohl ben Fleif́ ber Bienenvölker in ber Sjaupttracht Durch Brutbejchränkung herabzumindern als bamit zugleich auch noch bie innere $\mathfrak{B e r f a j i u n g}$ bes Biens fo zu jhäbigen, daß bie ichlimmiten Folgen nicht ausbleiben können.

Echließjlich weijen wir noch darauf hin, daß̉ bei der gejchilderten Betriebsmeije eine $\mathfrak{B e r m e h r u n g}$ bes Standes ausgejchlofien ijt und baj Durch eine gefonderte künftliche Röniginnenzucht und llmweifelung für bie $\mathfrak{B e r j u ̈ n g u n g ~ D e r ~ R o ̈ n i g i n n e n ~ g e j o r g t ~ m e r b e n ~ m u ß ́ , ~ w a s ~ m a n c h e m ~}$ Imker boch auch als ein jehr umitändliches $\mathfrak{B e r f a h r e n ~ e r j c h e i n t , ~ m i t ~ b e m ~}$ 
er keineswegs immer trog aller aufgemandten Zeit und Mühe gute Err= fahrungen gemacht hat. Führt man aber ben eben gejchilderten Betriebs= plan burch, jo muß man jchon bieje Umitändlichkeiten mit vielen anderen mit in Den Rauf nehmen.

Breū́ befindet fich eingeitandenermaß̧en in einiger $\mathfrak{B e r l e g e n h e i t , ~ a l s ~}$ er bie Frage Der $\mathfrak{B e r m e h r u n g}$ jeines Standes behandelt. Er empfiehlt

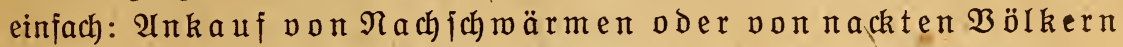
a us Der Syeide - ober einige Stöcke Des Standes als Schrarmitöcke zu behandeln, D. h. umgekehrt, wie er jelbjt jonjt empfiehlt, zu imkern. Und ein neuzeitlicher Bienenzüchter, Der von Rafiezucht etwas gehört hat, wird erít recht bedenklich mit Dem Ropfe jchütteln, menn er hört, baß̉ Preuß̧ Die Einführung Der ichwarmluftigen Seibebienen in Frühtracht= gegenden gutheijt, ja jogar empfiehlt, obgleich er kurz vorher bavor ge= warnt hat. $\mathfrak{E s}_{\text {j }}$ teckt eben ein grojer Rechenfehler in ber fonjt jo jharf= finnig ausgeklügelten Betriebsmeije von Preuß́ und allen jeinen Nachjolgern. Und auch mit Der Röniginnenzucht in $\mathfrak{B}$ ölkern ober mit $\mathfrak{B}$ ölkern, Die an Der Entfaltung ihrer Triebkraft abjichtlich gehindert morben find, hat es jeinen jchlimmen Şaken, wie uns jeder erfahrene Röniginnenzüchter zu= gejtehen wird, ohne dá̉ wir unjere Behauptung ausführlich zu begründen brauchen. Die Widernatürlichkeit Der Betriebsweije macht ihre Wirkungen eben auf allen ausjhlaggebenden Gebieten - und bazu gehört boch in eriter Sinie Die Röniginnenzucht! - geltend.

Lnd mun ftehen wir vor ber Enticheidungsfrage: Gibt es keinen anderen $\mathfrak{W e g}$ zum Ziel, Der bie Mängel bes gejchilderten und jachlich kritifiterten $\mathfrak{B e r f a h r e n s}$ vermeidet und auf einfachere und mehr natürliche Weije ohne Das 2bjteck= und Brutbejchränkungsmittel bennoch bie höchjten Sonigerträge erzielt? Wir verfahren tatjädlich anders und bie Tatjachen beweijen, dá̉ unjere Betriebsweijebei mindejtens gleichem Erfolge hinfichtlid bes Sonigertrages bod den Borzug hat, die Triebkräfte des Biens nicht zu unterorücken oder zu befchränken, jondern in volltem Maßje in unjere Dienfte zu itellen und auszunuben.

Um es gleich vorweg zu jagen: Wir jind nicht beherricht und be= jeijen von Der unheimlichen 2 ngit vor Dem unnüben Bienenfleijch in tracht= Iojer Zeit, melche Den 2rbjperrimkern ihre ganze Betriebsweije Diktiert hat und nach unjerer überzeugung unjere vaterländijche Bienenzucht nicht ge= fördert, fondern eher gejchäbigt hat, wie wir oben gezeigt haben.

Wer zwingt uns benn, bie unnü̈en Frefier in trachtlojer Zeit auf unje= rem Bienenjtande zu belaffen unto Den $\mathfrak{B}$ ölkern Das $\mathfrak{W i n t e r f u t t e r ~ w e g f r e f f e n ~}$ zu Iajfen. Gibt es Denn in unjerem $\mathfrak{B a t e r l a n d e}$ nur Begenden, in benen Mitte Suni alle Tracht vorüber ijt, gibt es nicht viele Gebiete, in benen Dann erjt die SJauptracht aus Weiphklee, Sinde, Buchweizen, Serradella und 
Sjeide beginnt und bann noch wochen= ja monatelang anbält. Gibt es nicht auch $\mathfrak{P o j t}$ und Eijenbahn, mittels beren man ben Bienenüberichú aus Frühtrachtgegenden nach Schlup der Iracht an bie Stellen bringen kann, mo bas in Frühtrachtgegenden unnübe Bienenfleifch in nüblichen Sjonig= ipeck verwandelt werben kann. (Bibt es nicht auch Bienenzüchtervereine, welche biefen neuzettlichen "Wanderbetrieb" in bie Sand nehmen und Durchführen können. Warum find wir denn fchwerfälliger als bic alten Ügnpter, melche fchon ber Reihe nach bie Ufer bẹs Nils durch ihre Bienen abgemeibet haben und wie unjere Syeidimker, welche ia alljährlich auch oft zmei= bis dreimal mandern, um die Saupttrachten auszumugen. Das ijt nun bas Ziel, welches wir fchon feit Jahren verfolgen, unmittelbar rach Schlǘ ber Faupttracht in Frühtrachtgegenden bie Bienen in foldhe Gegenden zu überführen, in benen dann gerade bie Iracht einjebt. Uuf bieje Weife könnten bie. überichü $=$ bienen aus Frühtrachtgegenden beffere $\mathfrak{B}$ erwertung finden und ungeheure Echäbe gehoben merben, bie heute zum gropen Teile ungenugt zugrunde gehen. Wir find überzeugt, daßj es an 2rbnehmern von Echwärmen und Feglingen aus Frühtrachtgegenden nicht fehlen mirb. Es ift ein verdienjt= Iiches Werk Des "थ̂lusfhuffes für Bienenkunde im Minifterium für \&and= mirtichaft in Berlin", Daß er bemüht ijt, Trachtkarten herauszugeben, $D$. h. nachzumeifen, wie die Trachtwerhältniffe in ben verichiedenen Gegenden be= fchaffen find und wohin es fich Iohnt, zu wandern. Dieje Irachtkarten können mit ber Zeit bie Grundlagen bilden für eine grob̈zügige Drganifation ber 2usnubung ber vorhandenen Trachtquellen burch Sinjchaffen von Bienen aus Gegenden, in benen bie Tracht früh verfiegt. 2uch bie Eifenbahnbeamten, welche fich jebt in jehr erfreulicher Weije ber edeln Bienenzucht in gropier Menge zumenden, könnten fich für bie थusführung bes Planes, bie Früh= trachtüberfchußjbienen in Spättrachtgegenden zu überführen, zur $\mathfrak{V e r f u ̈ g u n g ~}$ ftellen, indem fie überall an ben Strecken, wo Spättracht vorhanden iit, Wanderbienenjtände errichten und bajelbjt $\mathfrak{B}$ ölker zur 2usnubung Der Tracht aujitellen unter 2ufficht bes Streckenperionals. Das ijt aber bas

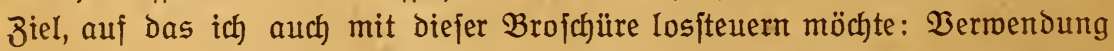
und 2นusnubung Der überichuß̉bienen aus Frühtrachtgegenden in Spättracht= gegenden. Wir haben barum nach ভchlufa der Iracht die $\mathfrak{B}$ ölker, welche nicht gejchwärmt haben, abgekehrt und bie jogenannten feglinge in genden und an Jmker verkauft, welche fie noch zweckmäpig verwenten konnten.

Der deutichen Jmkermelt mub doch ichließ̧lich ein doppeltes grob̉es Biel vorichmeben, nämlich bie gefamte vorhandene Iracht, Frïh=, ธommer = und Epätracht in ganz Deutfdland möglichft gründlichunderfchöpfendausunuben, um für unfere $\mathfrak{B}$ olks= ernährung ourch unfere $B$ ienen den ebeliten aller $\mathfrak{x a ̈ h r f t o f f e}$ 
aus der Pflanzenwelt, den Sonig, reftlos zu erfafien und iammeln zu lafien, dabei aber auch alle Lebenskräfte aller Bienenvölkeringanz Deut f hlandreftlosinden Dienjt biefer

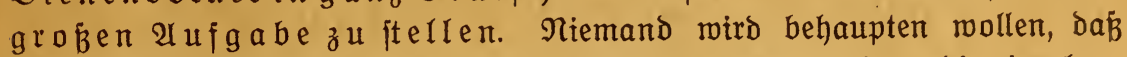
bie großßen $\mathfrak{B}$ erbände ber beutichen Imkerwelt, gejchmeige denn die einzelnen Jmker, bisher biejes hohe Zitel jich gejteckt oder mit Bemúptjein verjolgt oder gar erreicht haben. Der einzelne Jmker hat fich immer nur bemüht, bie Bientrmeide des Flugkreijes feines Bienenitandes auszumuken, un= bekümmert darum, daßj vielleicht dicht baneben ober boch in leicht erreich= barer Ferne reiche Trachtquellen vorhanden waren, bie von keinem Bienen= ftande ausgejchöpft wurben. 2Uber gar ben Wustaujch ber Bienen unter Den Bienenzüchtern in Frühtracht= und Spättrachtgegenden zu organijieren und burchzuführen, das will den meijten noch als eine lltopie, als ein undurchjührbarer Bedanke erjcheinen. Und boch ift bas ein jo einfacher

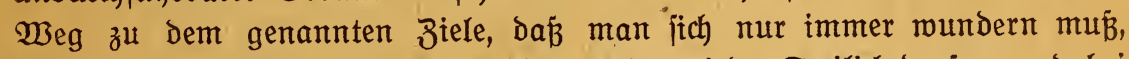
daß̧ er nicht betreten und energif nicht auf einem allzu engherzigen, egoijtifchen Standpunkte itehen und meinen, es jei viel belfer, überichuß̧bienen burch Unterbrücken und $\mathfrak{B} e=$ fchränken des Betriebes überhaupt nicht aufkommen zu lajien, als fie zu einem billigen Preije feinen Spättracht=Smkerbrübern zur 2 Usmubung ihrer Tracht zu überlajfen.

Um bie befiere 2 usnugung Der Spättracht Durch Die überichuß̈bienen aus Frühtrachtgegenden zielberwǚt Durchführen zu können, wäre es wünichens= mert, ১aß̉ die Spättrachtimker fich mehr dem Mobilbetrieb zumenden mürden, bamit jie bie anfangs Juli aus den Frïhtrachtgegenden ihnen zugejandten überjchuß̈bienen in Gejtalt von Schmärmen und, Feglingen auf aus= gebaute Waben merfen könnten, fo bá diejelben bis zu Beginn ber Epättracht aus Buchreizen ober Sjeibe jich in gewünjchter Berfajiung und auf höchjter Flugkraft befänden und auch bie Wanderung ficher überitehen mürben, ba fie nicht auf ganz jungem Wachje jäß̈en.

Würben bie $\mathfrak{B}$ ölker vom 1. bis 10. Jult zur 2ufftellung kommen, fo mürben bie Bienen aus dem Schmarmbrutjab ichon vom 28. bis 30 . Jult ausfchlüpfen und von Mitte 2Uuguft an, aljo vom Beginn ber Epättracht

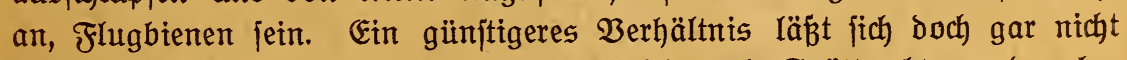
Denken, als es tatjächlich zwijchen ben Früh= und Spättrachtgegenden be=

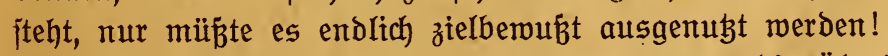

Es gibt auch noch einen gangbaren Weg, bie überjhuß̉bienen aus Jrühtrachtgegenden nubbringend zu verwenden und zugleich bie Spättracht auszubeuten: Wan fchlägt alle Echmärme und Feglinge in Frühtracht= gegenden felbit auf ausgebaute Waben und verkauft bieje honigarmen Vörker auf ganzem Bau an Spättrachtimker oder fchickt fie in Spättracht= gegenden, um Durch fie die Spättracht zu eigenem Nuben auszubeuten. 
2lm ভchluß́ der Spättracht nimmt man die $\mathfrak{B}$ ölker wieder zurück und benubt Bienen und Brut zur $\mathfrak{B e r j t a ̈ r k u n g ~ D e r ~ S t a n d o b ̈ l k e r ~ u n t e r ~} 2 \mathfrak{d b}=$ tötung Der alten Röniginnen, oder man jtellt bieje $\mathfrak{B}$ ölker auch auf, Damit fie im nächjten Gahre bie Frühtracht ausnuben follen. Benubt man jie zur $\mathfrak{B}$ eritärkung, fo kann man bamit auf allerbejte weife bie immerhin koitfpielige und umitändliche fpekulatipe Syerbjtfütterung erjeben, da man ia in ben organijch kräftigen jungen Bienen aus ber Spättracht in befierer Qualität Den $\mathfrak{B}$ ölkern Das gibt, was man burch fpekulatipe Serbjtfütterung zumeift mur in geringerer Qualität zu'erzielen vermag.

Selbjtwerítändlich kann man auch mit den Spättrachtimkern, denen man Schwärme und Feglinge oder $\mathfrak{B}$ ölker auf ausgebauten $\mathfrak{W a b e n}$ geliefert hat, ein 2lbkommen wegen Rücklieferung von $\mathfrak{B e r j t a ̈ r k u n g s m a t e r i a l ~}$ treffen. Uns kam es hier darauf an, zu zeigen, 'dą̧ es verjchiedene gang= bare Wege zu Dem genannten "groß̉en ziele gibt, bie ganze Tracht unjeres Reichsgebietes ebenjo, wie die ganze Rraft unjerer Bienen für unjere

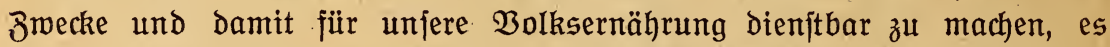
gilt eben nur, bä̉ bas groß̉e Jiel von allen beutichen Jmkern fcharf ins 2uge gefaj̧ wirb, und bie nachgemiefenen richtigen und auch gangbaren Wege befchritten werden. Dann bürfte auch bie "2l $\mathfrak{n g}$ itbienenzucht ", welche jebt Modejache in Deutjchland gemorden ift und unjere vaterländifche Bienenzucht fchwer jhäbigt, übermunden werben und für eine zielbemußjte Betriebsmeife unter 2usmubung aller Trachtquellen und aller Triebkraft ber Bienen fowohl in Früh= wie Spättrachtgegenden die $\mathfrak{B a h n ~ w i e d e r ~}$ jreigemacht jein.

Unjer Betriebsplan für die Bienenzucht in Frühtrachtgegenden läuft zunächjt parallel mit bem, welcher auch von Preub und anderen in fcharf= jinniger $\mathfrak{W e i j e}$ ausgebacht und bargejtellt worden ijt. Wir juchen auch zunächjt mit allen Mitteln die $\mathfrak{B o ̈ l k e r ~ u n m i t t e l b a r ~ v o r ~ d e r ~ S j a u p t t r a c h t ~}$ auf die SJöhe ber Flugkraft zu bringen und Dann während ber Sfaupt= tracht das Schwärmen zu verhüten. Uls Sjauptmittel wenden wir, wie ichon ermähnt, zulegt Den 2ustaujch auslaufender Brut gegen offene Brut aus jchmächeren $\mathfrak{B}$ ölkern an, haben aber auch nichts bagegen einzumenden, menn aus bejonderen (Bründen zur abjoluten $\mathfrak{B e r h u ̈ t u n g ~ d e s ~ Q u s j c h r w a ̈ r m e n s ~}$ ein fenkrechtes 2lbiperrgitter angemandt wirb, welches die Rönigin ant 2lusflug hindert, ohne fie jedoch in Der Eierlage zu bejchränken. Wir juchen ourch 2ustaujch ber auslaufenden und offenen Brutwaben auch eine Gleichmachung Der $\mathfrak{B o ̈ l k e ́ r ~ v o r ~ b e r ~ S ̧ a u p t t r a c h t ~ h e r b e i z u f u ̈ h r e n . ~ W 3 i r ~ f p e r r e n t ~}$ aber bie Rönigin nicht $\mathfrak{a b}$, noch bejchränken ober unterorücken wir aus eben Dargeftellten Gründen bie Brutentwicklung. Wir fuchen vielmehr ourch Die offene 3 rut Den Fleif3 Der Bienen in Der Sjaupttracht auf das थ̈ußzerite zu jteigern und nüben auch jonjt bie Triebkräfte bes Biens all= feitig fo jtark wie irgend möglich aus. Durch genilgenden Riaum zum 
Sonigaufipeichern und burch rechtzeitiges Schleudern fuchen wir ebenfalls bie Tätigkeit Des Biens aufs Schärfite anzuppannen. So nügen wir bie SFaupttracht mit bem ungeichmächten SJöchjtmaß ber $\mathfrak{B}$ olkskraft aus und feben alle Sjebel in Beriegung, um dies zu erreichen.

Wie jchon erwähnt, jegen wir Schwärme, welche gegen unjeren willen mährend ber Saupttracht fallen, auf ausgebaute Waben an bie Stelle ber Wutterftöcke und nuben auf bieje Weife Den Sammeltrieb erjt recht gründ= lich aus. Wir freuen uns, wenn dann am Schlǘ ber Tracht unjere $\mathfrak{B}$ ölker jtarke Schwärme abjtop̉en, welche wir, falls wir fie nicht zum Füllen von Sücken auf Dem eigenen Stande benötigen, verkaufen. Bei ben gegenwärtig hohen Bienenpreifen ijt bas ein Nuben aus ber Bienenzucht, ber uns nebenbei noch in ben Schoß fällt und nicht unbeträchtlich ijt.

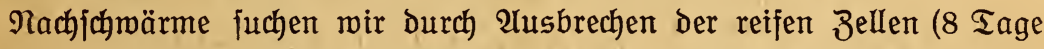
nach Dem Schwärmen) zu verhüten, Damit bie abgejchmärmten $\mathfrak{B}$ Ïlker recht jtark an $\dot{i} \mathfrak{u} \mathfrak{n} \in \mathbb{n}$ Bienen in die jpekulative Sjerbitfütterung eintreten, melche bann auch infolge ber jungen, fruchtbaren Rönigin vortrefflichen Erfolg hat und es bemirkt, dáa bie Stöcke in allerbejter $\mathfrak{V e r f a j f u n g ~ i n ~ D e n ~}$ Winter und in bas folgende Frühjahr kommen.

Was nicht jchroärmt, wird am Schlujie der Tracht abgefegt und ver= ichickt. Da es mun nicht ratjam ijt, abgefegte $\mathfrak{B}$ ölker jelbjt Röniginnen erziehen zu laffen, ijt es notwendig, rechtzeitig vorher und zwar in Der Saupttracht unter ben Denkbar günjtigiten $\mathfrak{V e r h a ̈ I t n i f f e n ~ R o ̈ n i g i n n e n ~ z u ~}$ züchten und befruchtet werden zu láfjen, welche Den abgefegten $\mathfrak{V}$ ölkern zugejebt werben können*). Reichen bieje Röniginnen nicht zu, jo läßjt man bie abgefegten $\mathfrak{B}$ ölker zunächjt Zellen anblajen und bedeckeln, zerjtört diejelben am fiebenten Tag und hängt Dann nochmals eine Wabe mit ganz jungen Maden aus einem bejonders guten Juchtvolke zu. Da jebt die $a b=$ gejegten $\mathfrak{B}$ ölker więer jehr viel junge Bienen bejiłen, fo erziehen fie gute Röniginnen. 2(cht Tage ipäter müfjen bie überjchüfifigen Zellen ausgebrochen merden, um ein Nachichwärmen zu verhindern.

2uf Dieje Weije wird nach ber Tracht auch eine Unterbrechung ber Bruttätigkeit in Den $\mathfrak{B}$ ölkern Durch Schwärmen ober 2 bjegen herbeigefühnt, welche in biejer Zeit aber nicht mehr jchäblich, jondern auß̈erordentlich nü̈blich wirkt. Sas während ber ganzen Frühjahrsentwicklung zu $\mathfrak{B r u t =}$ zwecken gebrauchte Brutnejt läuft einmal völlig aus, bie Zellen merden gründitch gereinigt, Mottenbrut zeritört uip. Dieje brutfreie ßeriode ijt Das alljährliche Gröreinemachen im Bienenjtock, welches unter lumjtänden jogar zur Entfernung von Faulbrutherden und von jonjtigen Rrankheits=

*) Eine vortreffliche Inleitung zu einer einfachen, volkstümlichen Röniginnen=

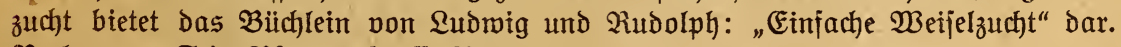
Verlag von Frib $\mathfrak{P f e n n i n g i t o r f f , ~} \mathfrak{B} e r l i n$ W 57. 
keimen führt. Deshalb möchten wir unjeren Bienen bieje "Babekur" nicht vorenthalten, zumal jie ja mit ber höchiten Rraftentfaltung bes Biens im Schwärmen verbunden ijt!

Ende Juni und anfangs Juli ift bieje allgemeine Säuberung ber Brutnejter und zugleich bie gründliche $\mathfrak{B}$ erjüngung ber $\mathfrak{B}$ ölker an STaupt und Błliedern vollzogen und bis zur ipekulativen Serbitfütterung find alle $\mathfrak{V}$ ölker wieder in vollem, regelrechtem $\mathfrak{B e t r i e b}$ und es wird bann ber (Grund gelegt für eine gute überwinterung und auch fchon für eine flotte (Ent= ricklung im folgenden Sahre. Damit aber fchlieft fich ber Rreis bes Betriebsjahres in Der Bienenzucht.

Seit Jahren haben wir uns vorgenommen, das Problem ber Bienen= zucht in Frühtrachtgegenden einmal für fich zu behandeln. Mangel an Zeit hat uns bisher baran gehindert. Wir hoffen, alle einfchlägigen Fragen jachlich bargejtellt und auch ben Lejer befähigt, zu haben, bie neu= zeitlichen Betriebsweijen felbjt nach ihren $\mathfrak{B}$ orzügen und Nachteilen richtig beurteilen zu können. Schließ̧lich haben wir unjere feit langen Jahrzehnten mohlerprobte Bienenpflege kurz vorgeführt, welche darauf hinausgeht, bie Saupttrachten mit ber Sauptkraft bes Bienenvolkes auszunuben, alle Triebe bes Bienenvolkes in Den Dienjt bes Jmkers zu ftellen und alle Naturmibrigkeiten möglichjt zu verhüten und zu vermeiden. Wir halten es auch in ber Bienenzucht in Frïhtrachtgegenden mit dem $\mathfrak{W o r t e , ~ m e l c h e s ~}$ wir btejer Brofchüre als Motto vorgejebt haben: "Fahret auf bie

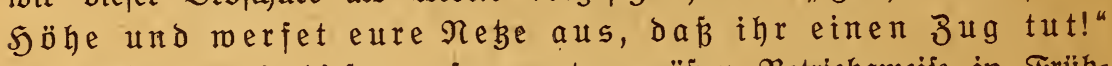
Und daßj wir mit biejer unjerer naturgemäßen Betriebsweije in Früh= trachtgegenden itets "Den reichjten $Z^{\text {ug }}$ " getan haben, dafür find bie $\mathfrak{E}=$ folge, bie wir und viele Taujende anderer Jmker in breiṕpigiähriger Praris mit inr erzielt und bie unjerer Betriebsmeife eine fo meite $\mathfrak{B e r}=$ breitung verjchafft haben, ficher ber bejte Tatbemeis, auf welchen wir auch unjere Sjoffmung gründen, baß̉ mit ber Zeit unjer rationeller Bienenzucht= betrieb in allen Frühtrachtgegenden fich einführen wirb. 


\section{Empfeblenswerte Schriften über Bienenz̨ucht.}

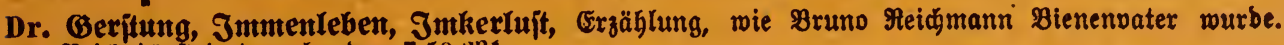
Feid ilujtriert., gebunden 7,50 纸.

Dr. Oexitung, Der Bien unb jeine 3udht. 5. vcrmehrte u. verbefferte Aruflage. Ungefähr 500 Seiten

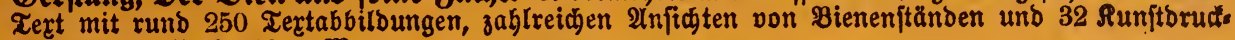
Bilbtafeln. \$reis 18,- $\mathbb{R}$.

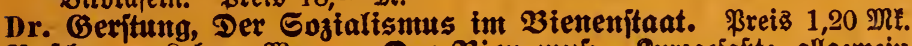

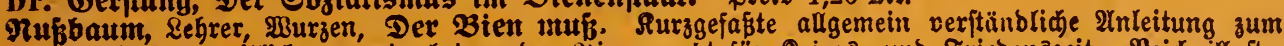

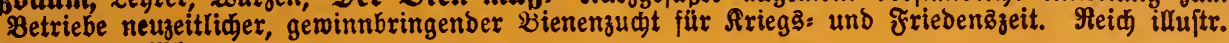
Preiz 2,- $\mathbb{M E}$.

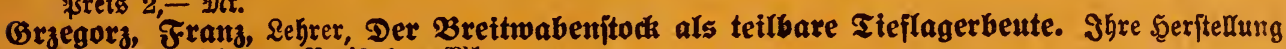
und : Behandlung. \$reiz 1,- Dit.

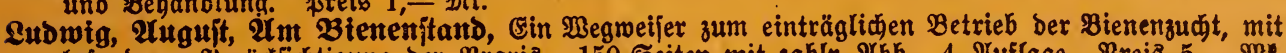

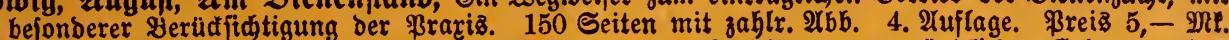

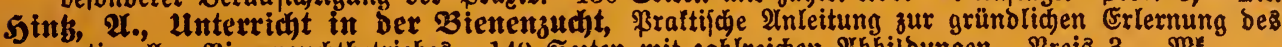

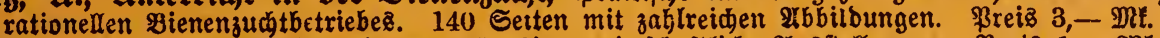

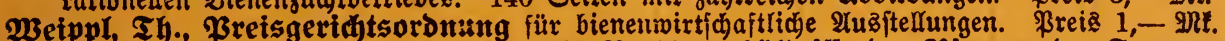

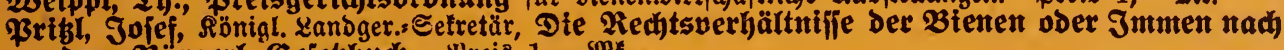

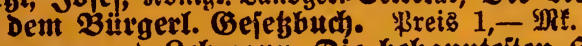

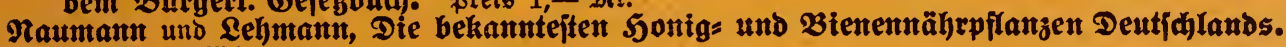
\$reißె 1,50 MRE.

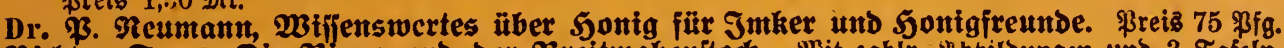

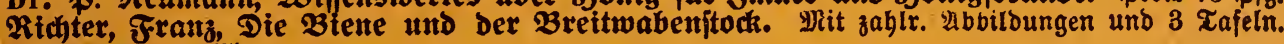
Breis 5,- บidt.

Dr. Rüftentadjer, Die Bienenkrankheiten nad ben neueften Forjoungen. \$reis 75 \$fg.

White, Die Urjadie ber europäijhen Faulbrut und ein Beridt über bie Eadibrut. überjęt von Dr. Sinitentadier. \$reiß 1,50 Mnt.

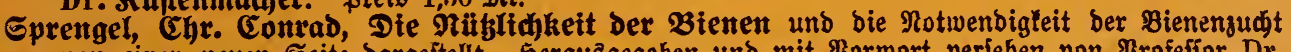
von einer neuen Seite bargeftellt. Derauggegeben uns mit Borwort verjeben von \$rofeffor Dr. 2ugujt Sirauje. Breiz 2,- Dif.

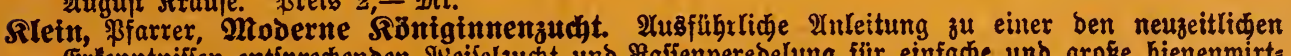

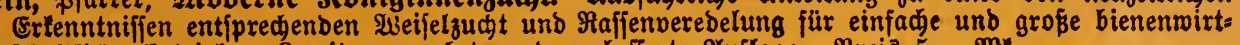
ichaftlide 2 etriebe. 3

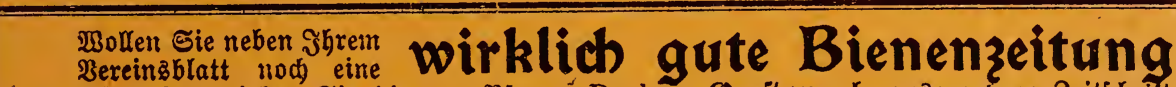 Iejen, fo beziehen Sie bie von \$iarrex Dr. h. c. Beritung berauzgegebene Zeitjwrift Die, deutiche Bienenzudt in Che eorie u. Praxis

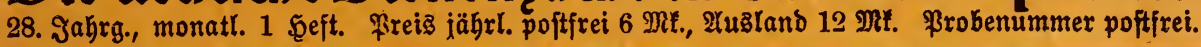

\section{Gute Büder über Sleintierzudt.}

unfer hausgrfiigel. I. Das 6rokgeflígel. Band 1: Raffekunde. Band 2: haltung, Pflege, Zunt und Oer" wertung. Dritte virmehrte und verbefierte Uuflage, neu bearbeitet von $\mathfrak{B}_{\text {. F }}$ Ieff $\mathrm{ner}$. Beibe Bände umfaffen zu. fammen ungefähr 800 Seiten mit rumb 500 Tertabbiloungen uno 50 farbigen und 32 fäwarzen Bilotafeln auf beftem Runitbrudkapier. Der \$reis für beide Bände in Salbleinen gebunden beträgt 60 Mk. - If. Die Caubenrallen.

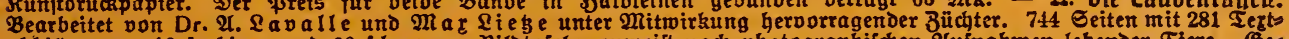
abbiloungen, 16 farbigen und 66 fa)marzen Bilotafeln, zumeift nach photographifden थufnahmen lebender Titere. (Be=

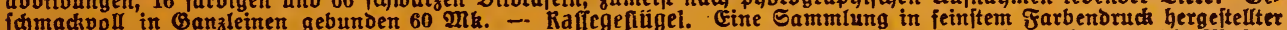
Standarbbilder nach Uquarellen von \&urt 3 an ber. 120 Blatt mit Dazugehörigem Tertheft, enthaltent Die Muiter:

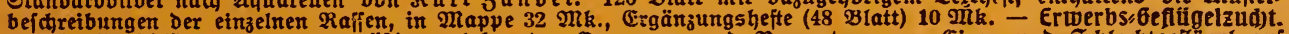

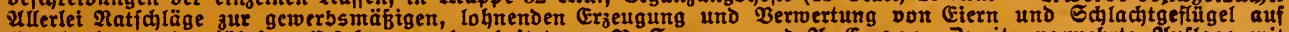

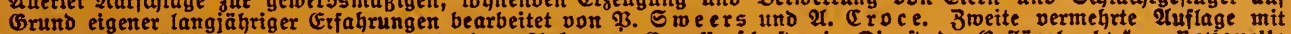

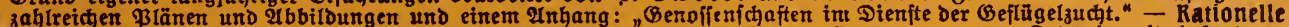

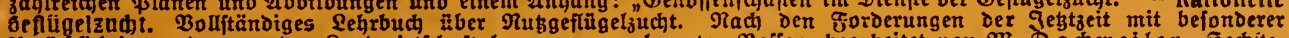

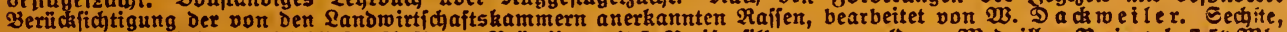

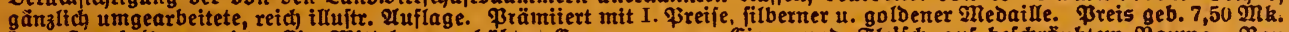

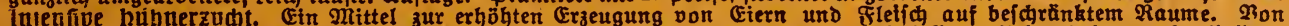

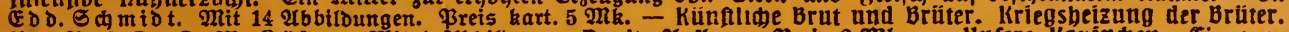

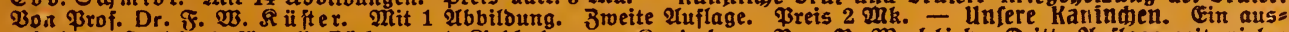

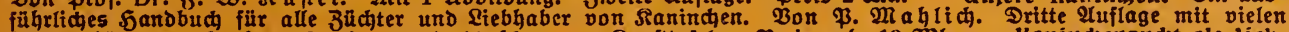

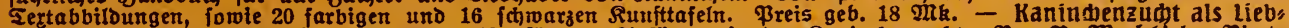

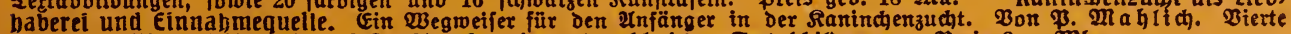

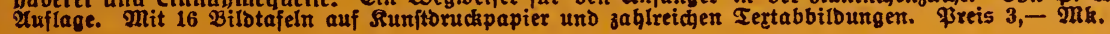




\section{Deutidhe \\ Bienenzudhtzentrale EDgar Geritung \\ Dỉmannitedt $\mathfrak{i}$. Ib.}

\section{Fabrikation und כeriand allex \\ Bienenzudhedarisartikel}

Spezłalität:

PFr. Dr. Geritungs Gnjtem

Thiir. Ständer $=\mathfrak{u n d}$ Sagerbenten in veridfiedenen 2 usfiuhrungen

Sonigidleudern, Runitwaben, Shiur. Sujtballons, 2bjperrgitter

Reid illuftriertes Sauptpreisbud auf Wumid 

DEMCO

\section{PAMPHLET BINDER}

Tan Pressboard. 
